\title{
Elastic and thermodynamical properties of cubic $(3 C)$ silicon carbide under high pressure and high temperature
}

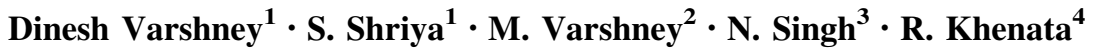

Received: 17 April 2015/Accepted: 15 July 2015/Published online: 5 August 2015

(C) The Author(s) 2015. This article is published with open access at Springerlink.com

\begin{abstract}
Pressure-dependent first-order phase transition, mechanical, elastic, and thermodynamical properties of cubic zinc blende to rock-salt structures in $3 C$ silicon carbide $(\mathrm{SiC})$ are presented. An effective interatomic interaction potential for $\mathrm{SiC}$ is formulated. The potential for $\mathrm{SiC}$ incorporates long-range Coulomb, charge transfer interactions, covalency effect, Hafemeister and Flygare type short-range overlap repulsion extended up to the second-neighbour ions, van der Waals interactions and zero point energy effects. The developed potential including many body non-central forces validates the Cauchy discrepancy successfully to explain the high-pressure structural transition, and associated volume collapse. The $3 C \mathrm{SiC}$ ceramics lattice infers mechanical stiffening, thermal softening, and ductile (brittle) nature from the pressure (temperature) dependent elastic constants behaviour. To our knowledge, these are the first quantitative theoretical predictions of the pressure and temperature dependence of mechanical and thermodynamical properties explicitly the mechanical stiffening, thermally softening,
\end{abstract}

Dinesh Varshney

vdinesh33@rediffmail.com

1 Materials Science Laboratory, School of Physics, Vigyan Bhavan, Devi Ahilya University, Khandwa Road Campus, Indore 452001, India

2 Department of Physics, M. B. Khalsa College, Indore 452002, India

3 Department of Physics, Ranchi College, Ranchi, Jharkhand 834008 , India

4 Laboratoire de Physique Quantique et de Modélisation Mathématique (LPQ3M), Département de Technologie, Université de Mascara, 29000 Mascara, Algeria and brittle/ductile nature of $3 C \mathrm{SiC}$ and still awaits experimental confirmations.

Keywords Carbide $\cdot$ High pressure Elastic properties . Mechanical properties . Thermal expansion

\section{Introduction}

Silicon carbide, (SiC), a high quality technical grade ceramics, possesses wide energy band gap, low density, high strength, low thermal expansion, high thermal conductivity, high hardness, high melting point, large bulk modulus, low dielectric constant, high elastic modulus, excellent thermal shock resistance, and superior chemical inertness. The IV-IV SiC compound possesses tetrahedral of $\mathrm{C}$ and $\mathrm{Si}$ atoms with strong bonds in the crystal lattice and the availability of wide variety of its polytypes with unique structural and electronic properties. SiC has been a subject of immense interest as it is a very hard and strong ceramics with its application in requiring high endurance, such as car brakes and clutches and ceramics plates in bulletproof vests. The high thermal conductivity coupled with low thermal expansion and high strength gives this material exceptional thermal shock resistant qualities. Ceramics $\mathrm{SiC}$ with little or no grain boundary impurities maintain strength at very high temperatures with no strength loss $[1,2]$.

The structural transition, mechanical and elastic properties under low and high pressures of $\mathrm{SiC}$ have attracted much interest in both experimental [3, 4] and theoretical [5-15] investigations to elucidate the pressure-dependent behaviour of polytype [cubic $(3 C)$, hexagonal $(6 H)$, and rhombohedral $(15 R)] \mathrm{SiC}$. The energy-dispersive X-ray 
diffraction with a diamond anvil cell has identified structural transformation in $3 C \mathrm{SiC}$ [zinc-blende (ZB) (B3) structure to the rock-salt $(B 1)$ structure $\approx 100 \mathrm{GPa}]$ with a volume collapse of about $20.3 \%$ [3]. The transition is reversible and the zinc-blende phase is recovered below $35 \mathrm{GPa}$ upon decompression. Furthermore, $6 H$ polytype $\mathrm{SiC}$ is found to be stable up to about $90.0 \mathrm{GPa}$. Later, shock compression experiments on $6 H \mathrm{SiC}$ show a firstorder phase transition into a sixfold coordinated rock-salt structure around $105 \pm 4 \mathrm{GPa}$ with a volume reduction of about $15 \pm 3 \%$ [4].

Structural and thermal stability as well as high-pressure behaviour of $3 C-\mathrm{SiC}$ has been described both by ab initio [5-7, 11, 14, 16] and molecular dynamics simulations [8, $10,15]$. Based on ab initio density functional calculations with the local-density approximation (LDA) show that the transition pressure of $3 C-\mathrm{SiC}$ is around $60 \mathrm{GPa}[\mathrm{ZB}$ to rock salt (RS)] [5-7, 11-14, 16]. Following Perdew-Wang generalized gradient approximation (GGA) for the exchange-correlation potential and the Troullier-Martins pseudopotentials; the transition pressure of $\mathrm{SiC}$ at about $63 \mathrm{GPa}$ is predicted $[6,12,13]$. Using the Troullier-Martins pseudopotentials and the LDA, the phase-transition pressure of $100 \mathrm{GPa}$ is also documented [7, 14]. It is noted that first-principles LDA calculations underestimate critical pressure for structural phase transition.

The constant-pressure molecular dynamics (MD) simulation retraces the reversible phase transformation [ $3 C$ to $\mathrm{RS}$ ] in $\mathrm{SiC}$ [8]. The first-principle calculations clearly demonstrate the structural transformation of $\mathrm{SiC}$ from a fourfold coordinated structure to sixfold coordinated structure under pressure. The phase transition from the zinc-blende phase to the RS phase is associated with a cubic to only one intermediate state as monoclinic unit cell transformation. Later on, the structural phase transition and mechanical properties of $\mathrm{SiC}$ from the $\mathrm{ZB}$ structure to the $\mathrm{RS}$ structure under pressure are investigated in detail by the first-principles plane-wave pseudopotential density functional theory method [9]. The results on the high-pressure elastic constants illustrate that the $\mathrm{ZB}$ structure $\mathrm{SiC}$ is found unstable when the applied pressure is larger than 126.6 GPa consistent with the experimental data and the molecular dynamics (MD) simulation results.

The molecular dynamics with effective interatomic interaction potential for $\mathrm{SiC}$ incorporating two-body and three-body covalent interactions is also proposed. The covalent characteristics $\mathrm{SiC}$ are described by the threebody potential using modified Stillinger-Weber form. The molecular dynamics method with the developed interaction potential is employed to investigate the structural and dynamical properties of crystalline $3 C$, amorphous, and liquid states of $\mathrm{SiC}$ for several densities and temperatures $[10,15]$. The phase stability of $\mathrm{SiC}$ under high pressure and behaviour of elastic constants with temperature is worth for microscopic understanding as well as technological applications.

The quantum computations based on density functional theory (DFT) as the full-relativistic version of the fullpotential augmented plane-wave plus local orbitals method $(\mathrm{FP}-\mathrm{APW}+10)$ are powerful techniques and have the advantage of elucidating the ground state properties not only for small atomic systems but also for large molecules [17]. Density functional theory with approximate local and semilocal density functionals with nonlocal and long-range Coulomb interactions are effective for dense molecules and materials as well as short-range interactions for soft matter, van der Waals complexes, and biomolecules [18].

The quantum mechanical calculations suggest a favourable phase transition into a RS structure with different transition pressure for $3 C \mathrm{SiC}$. However, these underestimate critical pressure for structural phase transition. We thus aimed at computing the pressure-induced phase transition of $3 C \mathrm{SiC}$ by formulating an effective interatomic potential. Note that the determination of ground state properties of IV-IV compounds with complex bonds based on lattice dynamical models is not easy. One needs to take care of experimental data with high accuracy and precision that accounts for low degree of freedom. The object of the proposed investigation is to develop a differential model as well to integrate the observed effect enabling useful prediction with reduced input experimental parameters.

The lattice dynamical models are useful in yielding both qualitative and quantitative information also with suggestive parameterization of the materials parameter. The density functional quantum calculations need precisely structural information as atomic positions and space groups. It also cares the value of volume around the experimental volume of the system. The successive iteration is thus made to determine the total energy corresponding to this volume. The thermal equation of state is needed to determine the ground state structural properties. It includes the lattice parameters within stable structure, the bulk modulus and pressure derivatives of elastic constants. The transition pressure is obtained by the common tangent between the two pressure-volume curves.

While exploring the ground state and anharmonic properties of simple and complex molecules, two major methods, one based on analytical form of cohesion with effective physical understanding and other based on expensive and time taking computational methods, are progressive. In the analytical models with two body interactions, the force constant deduced does not validate the Cauchy discrepancy. Incorporating many body interactions in the interaction potential with different cohesive energy forms is seen as the relevant potential which validates the Cauchy discrepancy. It is thus the major objective to seek the importance of both 
charge transfer interactions and covalent nature apart from zero point energy effects of group IV-IV cubic $\mathrm{SiC}$ in the interaction potential.

While discussing the mechanical and thermodynamical properties, the lattice dynamical model based on charge transfer interactions has been found successful [19-26]. The overlap repulsion potential (extended up to second-neighbour ions) is based on Hafemeister and Flygare [27]. The short-range interactions as the induced charge dipoledipole and charge dipole-quadruple (van der Waals) interaction are found to be successful to determine the cohesion in several alkaline-earth solids [28]. As far as the pressure (temperature) induced mechanical and elastic properties as hardness, ductile nature, mechanical stiffening and thermal softening of $\mathrm{SiC}$ ceramics is concerned, these are probably the first quantitative theoretical prediction of its kind and still awaits experimental confirmations. Deduced aggregate Young's modulus, compression and shear wave velocities are in agreement with the observed values.

The opportunities offered by $\mathrm{SiC}$ ceramics have, therefore, motivated large research efforts which in turn have addressed only structural transitions and elastic, thermal and thermodynamical properties are still lagging. The proposed studies are organized in the following sequence. We first discuss the key assumptions to develop an effective interatomic potential between a pair of ions. We then support them by physical arguments for ceramics $\mathrm{SiC}$ followed by an equation of state in "Computational methodology" section. The Slater-Kirkwood variational method is employed to determine the induced charge dipole-dipole and charge dipole-quadruple (van der Waals) coefficients keeping in mind that both ions ( $\mathrm{Si}$ and C) are polarizable. In sequence, we compute phase-transition pressures, the second-order aggregate elastic constants within the Shell model. Thus, the importance of long-range Coulomb with charge transfer interactions, covalent nature of bonds, charge dipole-dipole and charge dipolequadruple (van der Waals) interaction, and the short-range overlap repulsive interaction up to second-neighbour ions is validated. In "Results and discussion" section, we discuss the various elastic properties.

As regard the ceramics $3 C \mathrm{SiC}$, a number of works have been made on the structural stability of high-pressure phases but the pressure and temperature-dependent properties are sparse as normalized volume, aggregate secondorder elastic constants $C_{i j}$, Bulk modulus $B_{T}$, Cauchy discrepancy in second-order elastic constant $\Delta_{1}^{2}$, second-order elastic constant anisotropy $\gamma_{1}^{2}$, melting temperature $T_{\mathrm{M}}$, aggregate third-order elastic constants $C_{i j k}$, Cauchy discrepancy $\Delta_{i}^{3}$ in third-order elastic constant, third-order elastic anisotropy $\gamma_{i}^{3}$, isotropic shear modulus $G_{H}$, Voigt's shear modulus $G_{\mathrm{V}}$, Reuss's shear modulus $G_{\mathrm{R}}$, Young's modulus $E$, Poisson's ratio $v$ and Pugh ratio $\phi\left(=B_{T} / G_{H}\right)$ leading to ductility (brittleness), Lamé's constant $\lambda$ and $\mu$, and elastic wave velocity $v_{1}$ and $v_{\mathrm{s}}$.

The anharmonic effects on $\mathrm{SiC}$ lattice are further studied by investigating the Gruneisen parameter $\gamma_{\mathrm{G}}$, isothermal compressibility $\beta$, Debye temperature $\theta_{\mathrm{D}}$, hardness $H_{V}$, heat capacity $\mathrm{C}_{\mathrm{v}}$, and thermal expansion coefficient $\alpha_{\text {th.exp. }}$ either in ZB or RS structures. The relevant expressions are documented in Appendix 1 and 2. We also provide a comparison of computed values with observed experimental and other theoretical studies. The major conclusions are presented in "Concluding remarks" section.

\section{Computational methodology}

To study the phase stability and the aggregate elastic constants of $\mathrm{SiC}$ under high pressures, we formulate an interatomic potential. The proposed interaction potential is based on the following assumptions: variations in force constants of $\mathrm{Si}$ and $\mathrm{C}$ are small, the short-range interactions between $\mathrm{Si}$ and $\mathrm{C}$ atoms are effective up to their second-neighbour ions, and harmonic elastic forces are viable for a pair of atoms without any internal strains within the crystal. Application of mechanical pressure as external variable causes an increase in the overlap of adjacent ions in a crystal. The result is a charge transfer takes place between the overlapping electron shells of $\mathrm{Si}$ and $\mathrm{C}$ atoms. The transferred charges interact with neighbouring charges around the lattice via Coulomb's law. Also, the chemical bonds in $\mathrm{SiC}$ are both ionic and covalent in nature and the application of pressure causes change in crystal structure (first-order structural phase transition), volume collapse and elastic properties.

The proposed interatomic potential is thus a route to discuss the structural transitions, mechanical properties in particularly, about stiffness, softeness, ductile, brittle nature, elastic constants anisotropy, compression and shear elastic wave velocity. In addition, the potential is predictive to explain the validity of non-central forces and anharmonicity from thermodynamical properties namely Debye temperature, heat capacity and thermal expansion coefficient of $3 C \mathrm{SiC}$. At zero pressure and variable temperature, the underlined effective interatomic potential also describes some of the temperature-induced thermal and thermodynamical properties of $3 C \mathrm{SiC}$.

The effective interatomic potential at ambient pressure invokes an isolated stable phase when the crystal free energy is minimized for the specified thermodynamic conditions. For this purpose, if the variables such as temperature, pressure or magnetic field applied on the crystal are altered, either the free energy changes smoothly and continuously or discontinuously. Variations in free energy cause structural phase transition. The $\mathrm{SiC}$ ceramics transform from their initial $B 3$ to $B 1$ structure under pressure. 
The minimum Gibbs's free energy, $G$, infers the stability of a particular structure. Herein, Gibbs's free energy, $G=U+P V-T S$. The notations are: $U$ is internal energy, which at $0 \mathrm{~K}$ is the cohesive energy, $S$ is the vibrational entropy at absolute temperature $T$, pressure $P$ and volume $V$. The thermodynamically stable phase at a given pressure $P$ and at zero temperature is the one with lowest enthalpy. The thermodynamical potential is thus the Helmholtz free energy $(H)$.

The interatomic potential with pressure or temperature as thermodynamical variable requires the estimation of the Gibbs's free energies for $\mathrm{ZnS}(B 3)$ phase and $\mathrm{NaCl}(B 1)$ phase. We use Born equation: $G_{B 3}(r)=U_{B 3}(r)+3.08 P r^{3}$ for $\mathrm{ZnS}(B 3)$ phase and $G_{B 1}\left(r^{\prime}\right)=U_{B 1}\left(r^{\prime}\right)+2 P r^{\prime 3}$ for $\mathrm{NaCl}(B 1)$ phase. At phase-transition pressure $P$ and at zero temperature: $G_{B 1}=G_{B 3}$ [29]. The notations $U_{B 3}(r)$ infer the total potential energy of $\mathrm{ZnS}(B 3)$ phase and $U_{B 1}\left(r^{\prime}\right)$ as total potential energy for the RS $(B 1)$ phase. The unit cell volumes are $V_{B 3}\left(=3.08 r^{3}\right)$ and $V_{B 1}\left(=2 r^{\prime 3}\right)$. The nearest neighbour distance is $r\left(r^{\prime}\right)$ in ZB (RS) structure. The total potential energy for $\mathrm{ZnS}(B 3)$ and $\mathrm{NaCl}(B 1)$ phases is

$$
\begin{aligned}
U_{B 3}= & \left(-\alpha_{M} Z e^{2} / r\right)[Z+2 n f(r)]-C r^{-6}-D r^{-8} \\
& +n b \beta_{i j} \exp \left[\left(r_{i}+r_{j}-r_{i j}\right) / \rho\right] \\
& +\left(n^{\prime} b / 2\right)\left[\beta_{i i} \exp \left(\left(2 r_{i}-k r_{i j}\right) / \rho\right)\right. \\
& \left.+\beta_{j j} \exp \left(\left(2 r_{j}-k r_{i j}\right) / \rho\right)\right]+\left[\left\{\hbar<\omega^{2}>^{1 / 2} / 2\right]\right. \\
U_{B 1}= & \left(-\alpha_{M}^{\prime} Z e^{2} / r^{\prime}\right)\left[Z+2 m f\left(r^{\prime}\right)\right]-C r^{\prime-6}-D r^{\prime-8} \\
& +m b \beta_{i j} \exp \left[\left(r_{i}+r_{j}-r_{i j}^{\prime}\right) / \rho\right] \\
& +\left(m^{\prime} b / 2\right)\left[\beta_{i i} \exp \left(\left(2 r_{i}-k^{\prime} r_{i i}^{\prime}\right) / \rho\right)\right. \\
+ & \left.\beta_{j j} \exp \left(\left(2 r_{j}-k^{\prime} r_{j j}^{\prime}\right) / \rho\right)\right]+\left[\left\{h<\omega^{2}>^{1 / 2} / 2\right\}\right]
\end{aligned}
$$

Due to complex nature of bonds in $\mathrm{SiC}$, the ionic charge for $\mathrm{Si}$ and $\mathrm{C}$ atom cannot be determined uniquely. The calculation of the Madelung energy is thus modified by incorporating the covalency effects $[10,15,30,31]$. The charge in above equations is thus written incorporating the polarization of a spherical shaped dielectric in displacing the constituent positive ions. The charge transfer interactions caused by the deformation of the electron shells of the overlapping ions and the covalency effects are the major attributes of long-range Coulomb effects. The IV-IV semiconducting compound contains covalent bonds so that some electrons are distributed over the region between neighbouring atoms; in such situation the interaction energies are attributed from the contribution of charge dipoledipole and charge dipole-quadruple terms. The induced charge dipole-dipole and charge dipole-quadruple (van der Waals) interactions are the third and fourth terms which are the short-range vdW attractive potential energies.
The fifth and sixth terms are the short-range overlap repulsive energies. This is due to the overlap repulsion between $i j, i i$ and $j j$ ions. The Madelung constants for B3 (B1) phases are represented by $\alpha_{m}\left(\alpha_{m}{ }^{\prime}\right) . \beta_{i j}$ symbolized for Pauling coefficient and is defined as $\beta_{i j}=1+\left(Z_{i}\right)$ $\left.n_{i}\right)+\left(Z_{j} / n_{j}\right)$ with $Z_{i}\left(Z_{j}\right)$ and $n_{i}\left(n_{j}\right)$ as the valence and the number of electrons in the outermost orbit. The numbers of the nearest unlike $n(=4)$ and like $n^{\prime}(=6)$ neighbours are for $B 3(\mathrm{ZnS})$. Similarly, numbers of the nearest unlike $m(=6)$ and like $m^{\prime}(=6)$ are for $B 1(\mathrm{NaCl})$ structure. The $Z e$ is being the ionic charge, $k\left(k^{\prime}\right)$ is the structure factor for $B 3$ $(B 1)$ structures, and $b(\rho)$ is the hardness (range) parameters. We denote the nearest neighbour ion separations as $r\left(r^{\prime}\right)$ for $B 3(B 1)$ structures.

The last term in Eqs. 1 and 2 is the lowest possible energy of the system and is due to the zero point energy. Here, $<\omega^{2}>^{1 / 2}\left(=k_{B} \theta_{\mathrm{D}} / \hbar\right)$ is the mean square frequency related to the Debye temperature $\theta_{\mathrm{D}}$. The Debye temperature can be known either from Heat capacity measurements or from the Bulk modulus value using $\theta_{\mathrm{D}}=(\hbar /$ $\left.k_{B}\right) \sqrt{ }\left(5 r_{0} B_{T} / \mu\right)$. Herein, $r_{O}, B$ and $\mu$ are the equilibrium distance, Bulk modulus and reduced mass of the compounds. Henceforth, model potential for ground state incorporates the attractive, repulsive and zero point energy.

We use the variational approach to deduce the overall vdW coefficients $C$ (charge dipole-dipole) and $D$ (charge dipole-quadruple) [32]. The short-range vdW coefficients due to induced charge dipole-dipole and charge dipolequadruple interactions caused by $\mathrm{Si}$ atom and $\mathrm{C}$ atom are

$$
\begin{aligned}
& c_{i j}=\frac{3}{2} \frac{e \hbar}{\sqrt{m_{e}}} \alpha_{i} \alpha_{j}\left[\left(\frac{\alpha_{i}}{N_{i}}\right)^{1 / 2}+\left(\frac{\alpha_{j}}{N_{j}}\right)^{1 / 2}\right]^{-1} \\
& d_{i j}=\frac{27}{8} \frac{\hbar^{2}}{m_{e}} \alpha_{i} \alpha_{j}\left[\left(\frac{\alpha_{i}}{N_{i}}\right)^{1 / 2}+\left(\frac{\alpha_{j}}{N_{j}}\right)^{1 / 2}\right]^{2}\left[\left(\frac{\alpha_{i}}{N_{i}}\right)+\frac{20}{3}\left(\frac{\alpha_{i} \alpha_{j}}{N_{i} N_{j}}\right)^{1 / 2}+\left(\frac{\alpha_{j}}{N_{j}}\right)\right]^{-1} .
\end{aligned}
$$

$c_{i i}=\frac{3}{2} \frac{e \hbar}{\sqrt{m_{e}}} \alpha_{i} \alpha_{i}\left[\left(\frac{\alpha_{i}}{N_{i}}\right)^{1 / 2}+\left(\frac{\alpha_{i}}{N_{i}}\right)^{1 / 2}\right]^{-1}$,

$d_{i i}=\frac{27}{8} \frac{\hbar^{2}}{m_{e}} \alpha_{i} \alpha_{i}\left[\left(\frac{\alpha_{i}}{N_{i}}\right)^{1 / 2}+\left(\frac{\alpha_{i}}{N_{i}}\right)^{1 / 2}\right]^{2}\left[\left(\frac{\alpha_{i}}{N_{i}}\right)+\frac{20}{3}\left(\frac{\alpha_{i} \alpha_{i}}{N_{i} N_{i}}\right)^{1 / 2}+\left(\frac{\alpha_{i}}{N_{i}}\right)\right]^{-1}$

$c_{j j}=\frac{3}{2} \frac{e \hbar}{\sqrt{m_{e}}} \alpha_{j} \alpha_{j}\left[\left(\frac{\alpha_{j}}{N_{j}}\right)^{1 / 2}+\left(\frac{\alpha_{j}}{N_{j}}\right)^{1 / 2}\right]^{-1}$,

$d_{j j}=\frac{27}{8} \frac{\hbar^{2}}{m_{e}} \alpha_{j} \alpha_{j}\left[\left(\frac{\alpha_{j}}{N_{j}}\right)^{1 / 2}+\left(\frac{\alpha_{j}}{N_{j}}\right)^{1 / 2}\right]^{2}\left[\left(\frac{\alpha_{j}}{N_{j}}\right)+\frac{20}{3}\left(\frac{\alpha_{j} \alpha_{j}}{N_{j} N_{j}}\right)^{1 / 2}+\left(\frac{\alpha_{j}}{N_{j}}\right)\right]^{-1}$.

In the above equations, the notations: $m_{e}, e$ and $Z$ are mass of the electron, charge and valence of the 
constituent metallic element, respectively. The symbols $\alpha_{i}$, and $\alpha_{j}$ represent the polarizabilities of $i$ th and $j$ th ion, respectively. The effective number of electrons responsible for polarization is symbolized by $N_{i}$ and $N_{j}$. The lattice sums $S_{i j}$ and $T_{i j}$ enable one to compute the overall vdW coefficients $C$ and $D$ in terms of $c$ and $d$ values determined from Eqs. 1 to 8 . The lattice sums $S_{i j}$, and $T_{i j}$ are expressed as [28]:

$$
\begin{aligned}
& C=c_{i j} S_{i j}+c_{i i} S_{i i}+c_{j j} S_{j j} \\
& D=d_{i j} T_{i j}+d_{i i} T_{i i}+d_{j j} T_{j j}
\end{aligned}
$$

$\mathrm{SiC}$ is a tetrahedrally coordinated covalent material and the complex chemical bonding corroborate both ionic and covalent nature. The Coulomb interaction between ions of $\mathrm{Si}$ and $\mathrm{C}$ atoms leads to charge transfer interactions. Apart from this, the covalent character of bond bending and stretching also needs to be incorporated in the potential. Thus, the second term in Eqs. 1 and 2 is an algebraic sum of non-central many body forces as the charge transfer force parameter and the force parameter arise due to covalent nature i.e. $f(\mathrm{r})=f_{c t i}+f_{\text {cov }}$. The charge transfer between ions of $\mathrm{Si}$ and $\mathrm{C}$ atoms is denoted in terms of a force parameter $f_{c t i}$ and is expressed as [27, 33, 34]:

$f_{c t i}=f_{0} \exp (-r / \rho)$

Here, $r_{i}\left(r_{j}\right)$ is the ionic radii of $i(j)$ ion.

The complex chemical bonding in IV-IV semiconducting compounds infers $\mathrm{SiC}$ as partially ionic and partially covalent in bonding. The attractive forces due to covalent nature thus modify the charge and are now the effective charge. The polarization effects originate from changes in covalency due to $\mathrm{Si}-\mathrm{Si}, \mathrm{Si}-\mathrm{C}$, and $\mathrm{C}-\mathrm{C}$ interacting electric fields. The covalency term in the interaction potential is thus expressed as [30, 31]:

$f_{\operatorname{cov}}(r)=\frac{4 e^{2} V_{s p \sigma}^{2}}{r_{0} E_{\mathrm{g}}^{3}}$

Here, $V_{s p \sigma}$ represents the transfer matrix element between the outermost $p$ orbital and the lowest excited of $s$ state. The transfer energy of electron from $p$ to the $s$ orbital is denoted as $E_{\mathrm{g}}$. The effective charge $e_{S}^{*}$ of $\mathrm{SiC}$ is related with the number of electrons transferred to the unoccupied orbitals from its surrounding nearest neighbour. The electron density is thus $n_{c}=1-e_{S}^{*} / e$. Thus, in $\mathrm{SiC}$ for overlap distortion effect $e_{S}^{*} \neq e$. The transfer matrix element $V_{s p \sigma}$ and the transfer energy $E_{\mathrm{g}}$ are related to electron density as $n_{c} / 12 \cong V_{s p \sigma}^{2} / E_{\mathrm{g}}^{2}$. The effective charge $e_{\mathrm{S}}^{*}$ is thus

$\frac{V_{s p \sigma}^{2}}{E_{\mathrm{g}}^{2}}=\frac{1-e_{s}^{*}}{12}$
The transfer energy $E_{\mathrm{g}}$ is

$E_{\mathrm{g}}=E-I+(2 \alpha-1) \frac{e^{2}}{r}$

Here, $E$ is the electron affinity for $\mathrm{C}$ and $I$ is the ionisation potential of constituent atom.

The Szigeti effective charge $e_{\mathrm{S}}^{*}(=Z e)^{*}$ is written in terms of the optical static dielectric constant $\varepsilon_{0}$ and the high frequency dielectric constant $\varepsilon_{\infty}$ as [19-26]:

$e_{s}^{* 2}=\frac{9 \mu \omega_{T O}^{2}\left(\varepsilon_{0}-\varepsilon_{\infty}\right)}{4 \pi N_{k}\left(\varepsilon_{\infty}+2\right)^{2}}$

and

$\frac{e_{s}^{* 2}}{e^{2}}=\frac{9 V \mu \omega_{T O}^{2}\left(\varepsilon_{0}-\varepsilon_{\infty}\right)}{4 \pi e^{2}\left(\varepsilon_{\infty}+2\right)^{2}}$

The symbol $\mu$ is the reduced mass, $N_{k}$ is the number of atoms present per unit cell volume i.e. $N_{k}=1 / V, \omega_{\mathrm{TO}}$ is the long wavelength transverse optical phonon frequency. Thus, for $3 C \mathrm{SiC} e_{\mathrm{S}}^{*}$ deviates from $e$ and is attributed to covalent nature of $\mathrm{Si}-\mathrm{Si}, \mathrm{Si}-\mathrm{C}$, and $\mathrm{C}-\mathrm{C}$ bonds.

Usually materials document a transition when the thermodynamical potential relevant to the given ensemble of the lower pressure phase equals that for some other structure in the absence of any barrier. The low pressure phase becomes the stable phase above this coexistence pressure. After determining the stable phase we also compute the higher order elastic constants, their pressure derivatives and anisotropy. The Appendix 1 illustrates the essential equations for the higher order elastic constants and their pressure derivatives. With these understanding of interatomic potential in $\mathrm{SiC}$, we have four material parameters, namely, modified ionic charge, hardness, range, force parameter $\left[Z_{m}, b, \rho, f(\mathrm{r})\right]$. Values of them can be deduced from equilibrium conditions [35-42].

\section{Results and discussion}

The application of pressure, temperature and magnetic field probably transforms materials from one structure to another. The relative stability of two crystal structures requires an extremely accurate prediction. The interatomic interaction potential with charge transfer interactions caused by ions of $\mathrm{Si}$ and $\mathrm{C}$ atom and covalent nature of $\mathrm{Si}-\mathrm{Si}, \mathrm{Si}-\mathrm{C}$, and $\mathrm{C}-\mathrm{C}$ bonds are effective in studying the structural phase transitions and elastic properties of tetrahedrally coordinated ceramics $3 C \mathrm{SiC}$. We evaluate the phase transition pressure by computing the Gibbs free energy $G=U+P V-T S$ for the ZB and RS phases. We note that the Gibbs free energy is thus the enthalpy $H(=U+P V)$ at $T=0 \mathrm{~K}$.

While doing high-pressure experiments, the huge applied pressure causes a reduction of the material volume. 
The temperature variations during the experiments will normally produce much smaller changes in the relative stabilities of different phases. Thus, the Gibbs free energy at zero temperature, which is the enthalpy $H$, is measured. The thermodynamically stable phase of crystal at $0 \mathrm{~K}$ and at ambient pressure $P$ is the one with the lowest enthalpy. Thus, the zero-temperature theoretical calculations are valid with experiment. In a situation when temperature variations are large during experiment for certain materials the effects of finite temperature may be significant. With these assumptions, we investigate structural and elastic properties of $\mathrm{SiC}$ in an ordered way.

The thermodynamical potential $G$ or $H$ in SiC is computed involving modified ionic charge, hardness, range and charge transfer parameters $\left[Z_{m}, b, \rho, f(\mathrm{r})\right]$ as [35-42]:

$\left|\frac{\mathrm{d} U(r)}{\mathrm{d} r}\right|_{r=r_{0}}=0$

Also, the bulk modulus $\left(B_{T}\right)$ :

$\left|\frac{\mathrm{d}^{2} U(r)}{\mathrm{d} r^{2}}\right|_{r=r_{0}}=\left(9 k r_{0}\right)^{-1} B_{T}$

We first deduce vdW coefficients $C$ and $D$ involved in expressions (1) and (2) from the Slater-Kirkwood variational method [32], for $3 C \mathrm{SiC}$ ceramics material parameters, and are enlisted in Table 1. The charge dipole-dipole and charge dipole-quadruple vdW coefficients are influenced by electronic polarizabilities. The polarizability values have been obtained from least-squares fit of experimental refraction data using additive rule and a Lorentz factor of $4 \pi / 3[43,44]$. We consider that the $\mathrm{SiC}$ to be partially ionic and covalent to discuss their structural, mechanical, elastic and thermodynamical properties in a systematic manner.

As a next step, we use the experimental data on lattice constant $(a)$ [45], the bulk modulus $\left(B_{\mathrm{T}}\right)$ [46], ionic (Ze), effective charge $\left(e_{s}^{*}\right)$ and the second-order aggregate elastic constant $C_{12}\left(C_{44}\right)$ [47] for determining the material parameters. Deduced values of hardness $(b)$, range $(\rho)$ parameter, and non-central many body forces arising due to charge transfer $\left(f_{\text {cti }}\right)$ and covalency $\left(f_{\text {cov }}\right)$ for $3 C \mathrm{SiC}$ ceramics are illustrated in Table 1 . The effective charge $e_{\mathrm{S}}^{*}$ depends on the values of optical dielectric constant $\varepsilon_{\mathrm{s}}$ and the high frequency dielectric constant $\varepsilon_{\infty}$. The value of long wave length transverse optical phonon frequency $\omega_{\mathrm{TO}}$ is taken from [48] to have the effective charge $e_{\mathrm{S}}^{*}$ and hence the covalency contribution.

We then minimize the Gibbs's free energies $G_{B 3}(r)$ and $G_{B 1}\left(r^{\prime}\right)$ for the equilibrium interatomic spacing $(r)$ and $\left(r^{\prime}\right)$ to determine structural phase transition of $\mathrm{SiC}$. Table 1 shows the optimized values of equilibrium interatomic spacing in $B 3$ and $B 1$ phases. The Gibbs's free energy $G_{B 3}(r)\left[G_{B 1}\left(r^{\prime}\right)\right]$ as functions of pressure $(P)$ for $\mathrm{SiC}$ is
Table 1 Estimated and input crystal data: vdW coefficients $\left[c_{i i}, c_{i j}\right.$, $\left.c_{j j}, C, d_{i i}, d_{i j}, d_{j j}, D\right]$, lattice constant $\left(a_{0}\right)$, bulk modulus $\left(B_{\mathrm{T}}\right)$, secondorder elastic constant $C_{12}\left(C_{44}\right)$, optimized value of ionic radii $r_{i}\left(r_{j}\right)$, hardness $(b)$, range $(\rho)$, charge transfer parameter $f(r)$, equilibrium distance: $r_{O}(B 3) ; r_{O}^{\prime}(B 1)$, Gibbs's free energy: $G_{B 3}(r) ; G_{B 1}(r)$

\begin{tabular}{ll}
\hline Input parameters & $\mathrm{SiC}$ \\
\hline$c_{i i}\left(10^{-60} \mathrm{erg} \mathrm{cm}^{6}\right)$ & 28.76 \\
$c_{i j}\left(10^{-60} \mathrm{erg} \mathrm{cm}^{6}\right)$ & 0.71 \\
$c_{j j}\left(10^{-60} \mathrm{erg} \mathrm{cm}^{6}\right)$ & 0.047 \\
$C\left(10^{-60} \mathrm{erg} \mathrm{cm}^{6}\right)$ & 14.07 \\
$d_{i i}\left(10^{-76} \mathrm{erg} \mathrm{cm}^{8}\right)$ & 14.284 \\
$d_{i j}\left(10^{-76} \mathrm{erg} \mathrm{cm}^{8}\right)$ & 0.297 \\
$d_{j j}\left(10^{-76} \mathrm{erg} \mathrm{cm}^{8}\right)$ & 0.002718 \\
$D\left(10^{-76} \mathrm{erg} \mathrm{cm}^{8}\right)$ & 3.019 \\
$a_{0}(\AA)$ & $4.36[45]$ \\
$B_{T}(\mathrm{GPa})$ & $227.0[46]$ \\
$C_{12}(\mathrm{GPa})$ & $142.0[47]$ \\
$C_{44}(\mathrm{GPa})$ & $256.0[47]$ \\
$r_{i}(\AA)$ & 0.42 \\
$r_{j}(\AA)$ & 1.162 \\
$b\left(10^{-12} \mathrm{erg}\right)$ & 7.512 \\
$\rho\left(10^{-9} \mathrm{~cm}\right)$ & 3.29 \\
$f(r)\left(10^{-3}\right)$ & 5.267 \\
Equilibrium distance $(\AA) r_{O}(B 3)$ & 1.89 \\
Equilibrium distance $(\AA) r_{O}^{\prime}(B 1)$ & 2.11 \\
Gibb’s free energy $\left(\mathrm{kJ} \mathrm{mol}^{-1}\right) G_{B 3}(r)$ & -4570 \\
Gibb’s free energy $\left(\mathrm{kJ} \mathrm{mol}^{-1}\right) G_{B I}(r)$ & -4498 \\
\hline
\end{tabular}

discerned in Fig. 1a. At zero pressure, the Gibb's free energy for $\mathrm{SiC}$ in $B 3$ crystal phase is more negative. Thus, at zero pressure $\mathrm{SiC}$ in $B 3$ phase is thermodynamically and mechanically stable, while the $B 1$ is not. On the other hand, above the phase transition pressure $\left(P_{T}=90 \mathrm{GPa}\right)$, the Gibb's free energy for $B 1$ system becomes more negative than $B 3$ phase, implying $B 1$ phase will be more stable. The cohesive energy per particle is obtained as $6.301 \mathrm{eV}$ for $3 C \mathrm{SiC}$ which is consistent with earlier experimental value of $6.34 \mathrm{eV}$ [49]; $7.415 \mathrm{eV}$ from ab initio density functional calculations and molecular dynamics method yields $6.3410868 \mathrm{eV}$ for $\mathrm{SiC}$ [50].

The phase stability of cubic $3 C \mathrm{SiC}$ under high pressures is essentially based on material parameters namely hardness $(b)$, range $(\rho)$, non-central many body forces as charge transfer force $\left(f_{\mathrm{cti}}\right)$ and covalency parameter $\left(f_{\text {cov }}\right)$. These are obtained from the experimental data. We comment that the available data based on one kind of experiment depend on the conditions of measurement. Henceforth, while developing a theory, one faces certain complications and one need to find suitable data that varies from technique to technique. For $\mathrm{SiC}$, we pay special attention while 
Fig. 1 Variation of Gibb's free energy for $B 3$ and $B 1$ phases with pressure and normalized volume with pressure and temperature
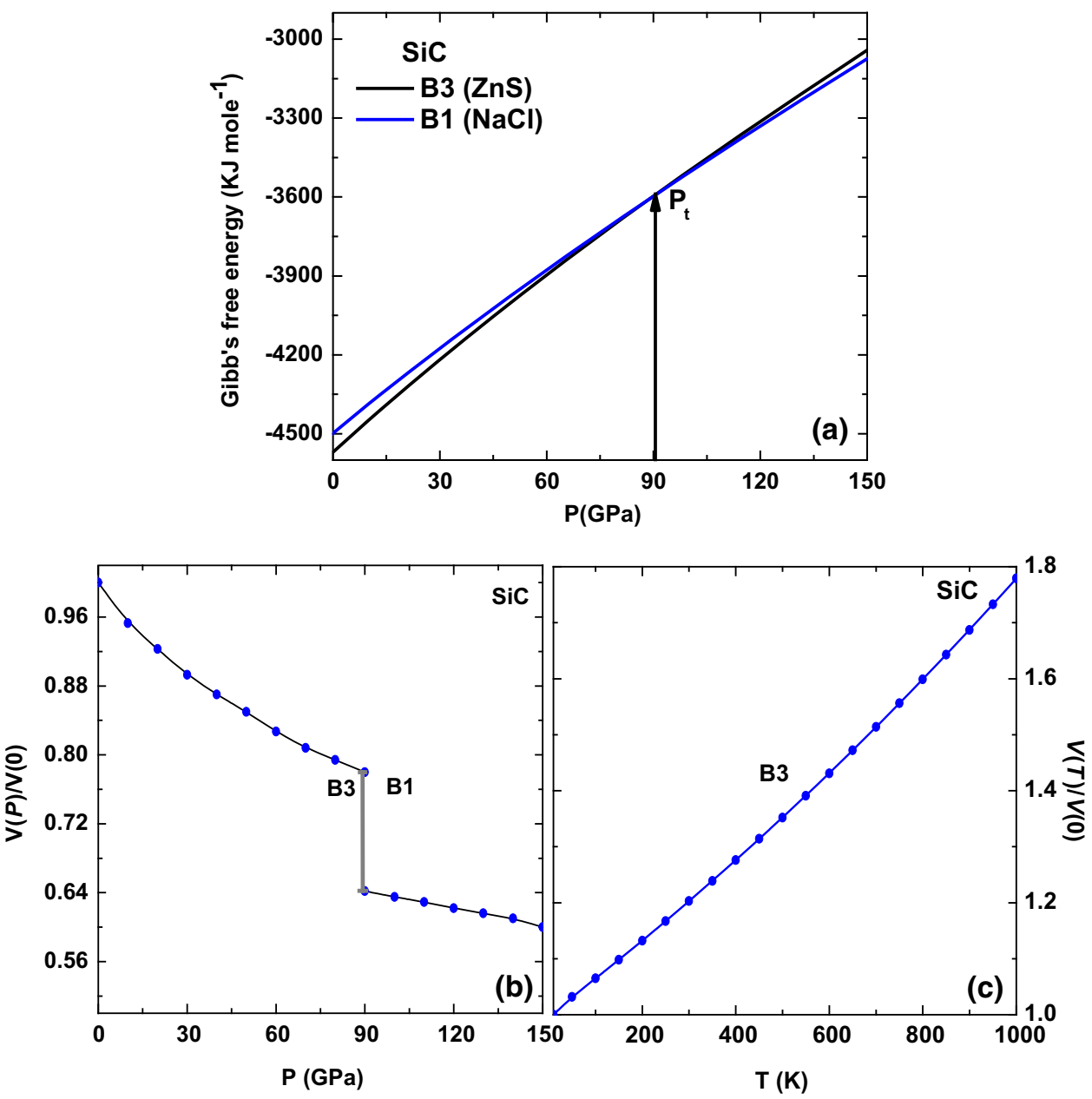

formulating interatomic potential to ensure whether long range or short-range interactions are at the origin of the structural transition. The many body force parameter as charge transfer $f(\mathrm{r})$ is positive as seen in Table 1 . It is attributed to the fact that the charge transfer parameter is computed from the difference of second-order elastic constants $C_{12}$ and $C_{44}$. The Cauchy energy $C_{12}-C_{44}$ is negative for SiC. Thus, consistent results lead to a test of the validity of assumptions made in model potential for $\mathrm{SiC}$.

$\mathrm{SiC}$ shows a crystallographic transition from $B 3$ to $B 1$ in certain pressure range. Table 2 illustrates the computed phase-transition pressure $\left(P_{T}\right)$ and compared with available experimental data $[3,4]$ and theoretical results [5-12, 1416]. $P_{T}$ for $\mathrm{SiC}$ is consistent with the experimental and other reported values and is attributed to proper formulation of interatomic potential, which considers the various interactions, explicitly the non-central many body forces as charge transfer interactions and covalency effects, as well as use of materials parameter based on experimental data. We may comment that any computational technique has its own limitations related to the chosen materials basic parameters, basic sets, as well the accuracy and precisions used apart from the approximations laid in the method. Needless to suggest that there is always a variation in estimated parameters by each technique.

The values of relative volumes associated with various compressions are estimated from [51]

$\frac{V_{P}}{V_{0}}=\left(1+\frac{B^{\prime}}{B_{0}} P\right)^{-1 / B^{\prime}}$,

Here, $V_{O}\left(B_{O}\right)$ is the cell volume (bulk modulus) of SiC at ambient conditions and $V_{P}$ is at finite pressure. The symbol $B^{\prime}$ is the pressure derivative of the bulk modulus. Figure $1 \mathrm{~b}$ documents the estimated value of pressure-dependent radius for both $B 3$ and $B 1$ structures, the curve of volume collapse $\left(V_{P} / V_{0}\right)$ with pressure to show phase diagram for $3 C \mathrm{SiC}$. The phase diagram will let us estimate the magnitude of the discontinuity in volume at the transition pressure. The value of relative volumes is shown in Table 2. It is also compared with various experimental [3, 4] and other theoretical works [5-9, 11-14, 16]. 
Table 2 Calculated transition pressure $P_{T}(\mathrm{GPa})$, volume collapse (\%), aggregate second-order elastic constants $\left(C_{11}, C_{12}\right.$ and $\left.C_{44}\right)$, aggregate bulk modulus $\left(B_{T}\right)$, (all are in $\left.10^{10} \mathrm{Nm}^{-2}\right)$ and pressure derivatives of SOECs $\left(\mathrm{d} B_{T} / \mathrm{d} P, \mathrm{~d} C_{44} / \mathrm{d} P\right.$ and $\mathrm{d} C_{S} / \mathrm{d} P$ ) for Silicon Carbide in $B 3$ phase

\begin{tabular}{|c|c|c|c|c|c|c|c|}
\hline Property & Present & Expt. & FP LMTO & LDF & LDA & MD & DFT \\
\hline Transition pressure $P_{T}$ & 90.0 & $\begin{array}{l}100[3], \\
105 \pm 4[4]\end{array}$ & & & $\begin{array}{l}66 \pm 5[5,11] \\
63[6]\end{array}$ & $\begin{array}{l}100[8] \\
90[10,15]\end{array}$ & $\begin{array}{l}66.6[16] \\
100[7,14] \\
75.4[9]\end{array}$ \\
\hline Volume collapse & 13.8 & $\begin{array}{l}20.3[3] \\
15 \pm 3[4]\end{array}$ & & & $18.5[5,11]$ & $21[8]$ & $\begin{array}{l}18.5[16] \\
12[7,14] \\
18[9]\end{array}$ \\
\hline$C_{11}$ & 37.11 & $\begin{array}{l}39.0[47] \\
36.3[56]\end{array}$ & $\begin{array}{l}42.0[51] \\
35.23[58]\end{array}$ & & $\begin{array}{l}44.9[62,64] \\
43.6[63]\end{array}$ & $\begin{array}{l}39.0[8] \\
39.0[10,15]\end{array}$ & $\begin{array}{l}41.51[9] \\
37.1[56]\end{array}$ \\
\hline$C_{12}$ & 22.34 & $\begin{array}{l}14.2[47] \\
15.4[56]\end{array}$ & $\begin{array}{l}12.6[51] \\
14.04[58]\end{array}$ & & $\begin{array}{l}14.6[62,64,65] \\
12.0[63]\end{array}$ & $\begin{array}{l}14.4[8] \\
14.26[10,15]\end{array}$ & $\begin{array}{l}13.19[9] \\
16.9[56]\end{array}$ \\
\hline$C_{44}$ & 27.93 & $\begin{array}{l}25.6[47] \\
14.9[56]\end{array}$ & $\begin{array}{l}28.7[51] \\
23.29[58]\end{array}$ & & $\begin{array}{l}25.6[62,64,65] \\
25.5[63]\end{array}$ & $\begin{array}{l}17.9[8] \\
19.11[10,15]\end{array}$ & $\begin{array}{l}26.54[9] \\
17.6[56]\end{array}$ \\
\hline$B_{T}$ & 27.30 & $\begin{array}{l}22.7[46] \\
22.4[59] \\
22.5[60]\end{array}$ & $\begin{array}{l}22.3[51] \\
21.1[58]\end{array}$ & $\begin{array}{l}24.9[34] \\
22.5[61]\end{array}$ & $\begin{array}{l}21.2[5,11] \\
22.5[62-65]\end{array}$ & $\begin{array}{l}22.5[8] \\
22.52[10,15]\end{array}$ & $\begin{array}{l}23.5[7,14] \\
22.71[9] \\
20.0[57] \\
22.5[56]\end{array}$ \\
\hline$C_{S}$ & 7.38 & & & & & & \\
\hline $\mathrm{d} B_{T} / \mathrm{d} P$ & 5.188 & $3.57[46]$ & $3.8[51]$ & $3.2[62,64,65]$ & $3.7[5,11]$ & $5.5[10,15]$ & $\begin{array}{l}3.79[9] \\
7.3[57]\end{array}$ \\
\hline $\begin{array}{l}\mathrm{d} C_{44} / \mathrm{d} P \\
\mathrm{~d} C_{S} / \mathrm{d} P\end{array}$ & $\begin{array}{c}5.198 \\
-0.0716\end{array}$ & & & & & & \\
\hline
\end{tabular}

Compressions in $\mathrm{SiC}$ at higher pressure indicate the mechanical stiffening of lattice.

Figure $1 \mathrm{c}$ discerns the variation of $V_{T} / V_{0}$ as functions of temperature in $B 3$ phase. Here, $V_{T}$ symbolizes the volume at various temperatures and $V_{0}$ at zero temperature and zero pressure volumes, respectively. A steep increase in the ratio $V_{T} / V_{0}$ with increasing temperature infers expansion of $\mathrm{SiC}$ lattice and is susceptible to temperature. On the other hand, $\mathrm{SiC}$ is compressed at higher pressures as shown in Fig. 1b. Henceforth, SiC lattice is thermally softened and mechanical stiffened. The normalized volume $V_{T} / V_{0}$ dependences on temperature are not known for $\mathrm{SiC}$, but the present behaviour is consistent with available experimental [52] and theoretical [53] data on $\mathrm{Li}_{2} \mathrm{O}$.

The response of any material that undergoes stress, deforms and then recovers and returns to its original shape after stress ceases leads to the determination of elastic properties. The elastic properties are vital in generating information about the binding characteristic between adjacent atomic planes, anisotropic character of binding and structural stability. Apart from the structural stability of $\mathrm{SiC}$ in $\mathrm{ZnS}(B 3)$ and $\mathrm{NaCl}(B 1)$ structures, we now compute the aggregate elastic constants at normal and under hydrostatic pressure. Deduced values are documented in Table 2.
Using the stress-strain coefficients, one determines the second-order aggregate elastic constants $C_{i j}$ under hydrostatic pressure with respect to finite strain. Also, proper parametrization of Coulomb, non-central many body forces, overlap repulsion, van der Waals interactions and zero point energy terms are essentially required.

The cubical symmetry of SiC dealt with three independent elastic constants $C_{i j}$. $C_{11}$ is a response of resistance to deformation by a stress applied on $(1,0,0)$ plane with polarization in the direction $<100>$. $C_{11}$ probes elasticity in length and a longitudinal strain produces a change in $C_{11}$. $C_{44}$ refers to the measurement of resistance to deformation with respect to a shearing stress applied across the (100) plane with polarization in the $<010>$ direction. $C_{12}$ and $C_{44}$ are related to the elasticity in shape, which is a shear constant. A transverse strain causes a change in shape without a change in volume and hence $C_{12}$ and $C_{44}$ are less sensitive of pressure as compared to $C_{11}$.

The variation of three independent second-order aggregate elastic constants (SOECs): $C_{11}, C_{12}$, and $C_{44}$ with external pressure for $\mathrm{SiC}$ in $B 3$ and $B 1$ phase is first discussed. As seen from Fig. 2 a that $C_{11}$ and $C_{12}$ increase with increase in pressure in both $B 3$ and $B 1$ phases. Also, $C_{44}$ decreases with the increase of pressure away from zero till the phase-transition pressures and then increases in $B 1$ 
Fig. 2 Variation of aggregate second-order elastic constants $\left(C_{i j}\right)$ with pressure and temperature

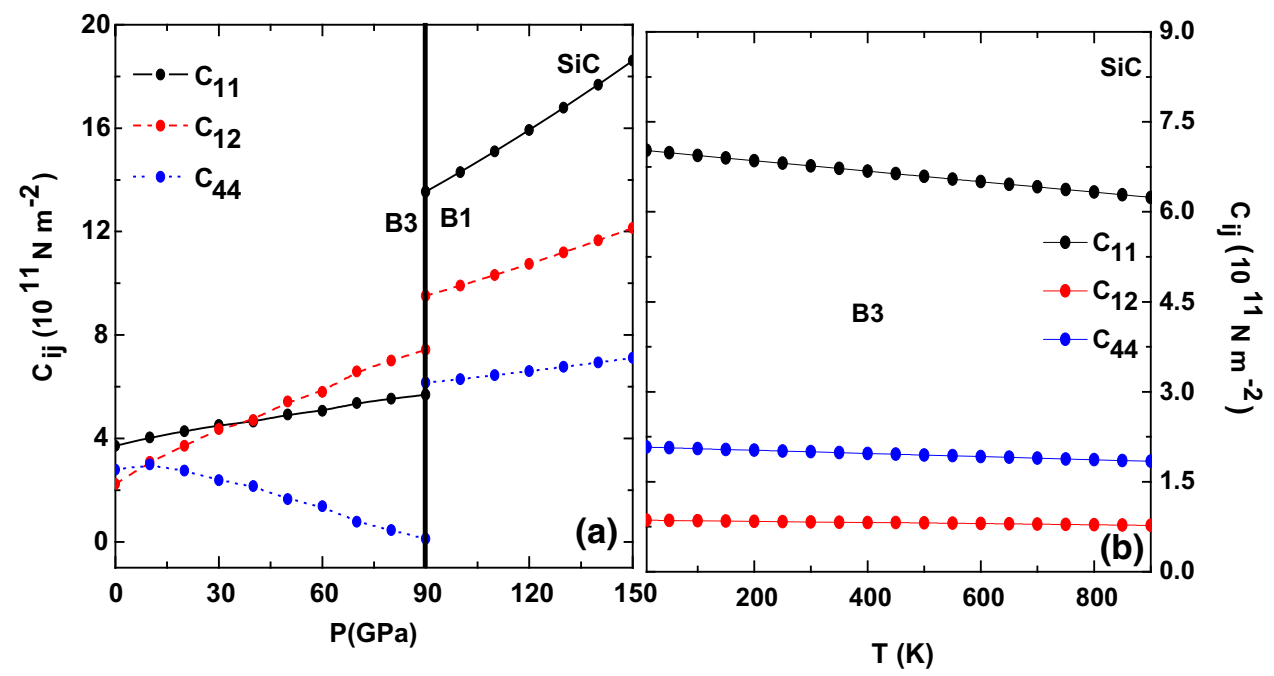

$B_{T}=\left(C_{11}+2 C_{12}\right) / 3>0$,

$C_{11}, C_{44}>0$,

and

$C_{S}=\left(C_{11}-C_{12}\right) / 2>0$.

Here, $C_{i j}$ are conventional aggregate elastic constants and $B_{T}$ is bulk modulus. We represent, $C_{44}$ and $C_{S}$ as the shear and tetragonal moduli of a cubic crystal.

Table 2 illustrates the computed values of bulk modulus $\left(B_{T}\right)$, shear moduli $\left(C_{44}\right)$ and tetragonal moduli $\left(C_{S}\right)$ which validates the elastic stability criteria for $3 C \mathrm{SiC}$ in $B 3$ phase. The second-order elastic constants critically depend upon pressure leading to $C_{12}-C_{44} \neq 0$. The mechanically stable phases for cubic crystal satisfy the Born criteria: $C_{12}-C_{44}>0$. The validity of above is readily seen by referring to Eqs. 51 and 52 for $C_{12}$ and $C_{44}$. The equilibrium condition leads to $B_{1}+B_{2}=-1.261 Z_{m}^{2}$ with emphasis on charge transfer interactions as well as covalency effects. For optimized values of $r_{i}\left(r_{j}\right)$ the Cauchy discrepancy $C_{12}-C_{44}$ is nonzero at zero pressure and at zero temperature. It is also valid when the many body noncentral forces are not involved in long-range forces. The short-range and long-range effects are naturally of similar order of magnitude. This is due to the fact $C_{i j}$ are calculated at optimized values of equilibrium distances rather than at experimental values.

Table 2 illustrates the calculated values of pressure derivatives of aggregate second-order elastic constants $\left(\mathrm{d} B_{T} / \mathrm{d} P, \mathrm{~d} C_{44} / \mathrm{d} P\right.$ and $\left.\mathrm{d} C_{S} / \mathrm{d} P\right)$. These are compared with available experimental $[46,47,56]$ and theoretical studies [8-10, 15, 46, 57-65]. For mechanical stability, the shear elastic constant $C_{44}$ is nonzero and is known by combining mechanical stability with minimum energy conditions. The high-pressure stability also suggests that the stable phase of elastic constants of a cubic crystal are as follows [29], 
Table 3 Calculated second-order elastic constant Cauchy discrepancy $\left(\Delta_{1}^{2}\right)$, anisotropy parameter $\left(\gamma_{i}^{2}\right)$, isotropic shear modulus $\left(G_{H}\right)$, Voigt's shear modulus $\left(G_{\mathrm{V}}\right)$, Reuss's shear modulus $\left(G_{\mathrm{R}}\right)$, Young's modulus $(E)$, Poisson ratio $(v)$, compressibility $(\beta)$ and Gruneisen parameter $\left(\gamma_{\mathrm{G}}\right)$ of Silicon Carbide in $B 3$ phase at zero pressure

\begin{tabular}{|c|c|c|c|c|c|c|c|}
\hline Property & Present & Expt. & FP LMTO & LDF & LDA & MD & DFT \\
\hline$\gamma_{1}^{2}\left(10^{10} \mathrm{Nm}^{-2}\right)$ & -0.736 & & & & & & \\
\hline$\Delta_{1}^{2}\left(10^{10} \mathrm{Nm}^{-2}\right)$ & -5.588 & & & & & & \\
\hline$G_{H}\left(10^{10} \mathrm{Nm}^{-2}\right)$ & 16.47 & $19.2[60]$ & $\begin{array}{l}21.9[50] \\
16.9[58]\end{array}$ & & & $12.37[10,15]$ & $14.1[56]$ \\
\hline$G_{V}\left(10^{10} \mathrm{Nm}^{-2}\right)$ & 19.71 & & $\begin{array}{l}23.1[50] \\
18.2[58]\end{array}$ & & & & $14.6[56]$ \\
\hline$G_{R}\left(10^{10} \mathrm{Nm}^{-2}\right)$ & 13.22 & & $\begin{array}{l}20.8[50] \\
15.7[58]\end{array}$ & & & & $13.6[56]$ \\
\hline$E\left(10^{10} \mathrm{Nm}^{-2}\right)$ & 41.22 & $44.8[60]$ & $\begin{array}{l}49.60[50] \\
40.1[58]\end{array}$ & & $\begin{array}{l}56.7[62,64,65] \\
55.0[62,64,65]\end{array}$ & $31.36[10,15]$ & $35.2[56]$ \\
\hline$v$ & 0.249 & $\begin{array}{l}0.267[47] \\
0.168[60]\end{array}$ & $\begin{array}{l}0.146[50] \\
0.201[58]\end{array}$ & & & $0.268[10,15]$ & $\begin{array}{l}1.0[5,11] \\
0.259[56]\end{array}$ \\
\hline $\begin{array}{l}\beta\left(10^{-11} \mathrm{~Pa}^{-1}\right) \\
\gamma_{\mathrm{G}}\end{array}$ & $\begin{array}{l}0.024 \\
1.015\end{array}$ & & & $1.01[61]$ & $1.12[5,11]$ & & $0.1518[5,11]$ \\
\hline
\end{tabular}

Fig. 3 Variation of Cauchy discrepancy $\left(\Delta_{1}^{2}\right)$ and elastic anisotropy $\left(\gamma_{1}^{2}\right)$ in second-order elastic constant $\left(C_{i j}\right)$ with pressure

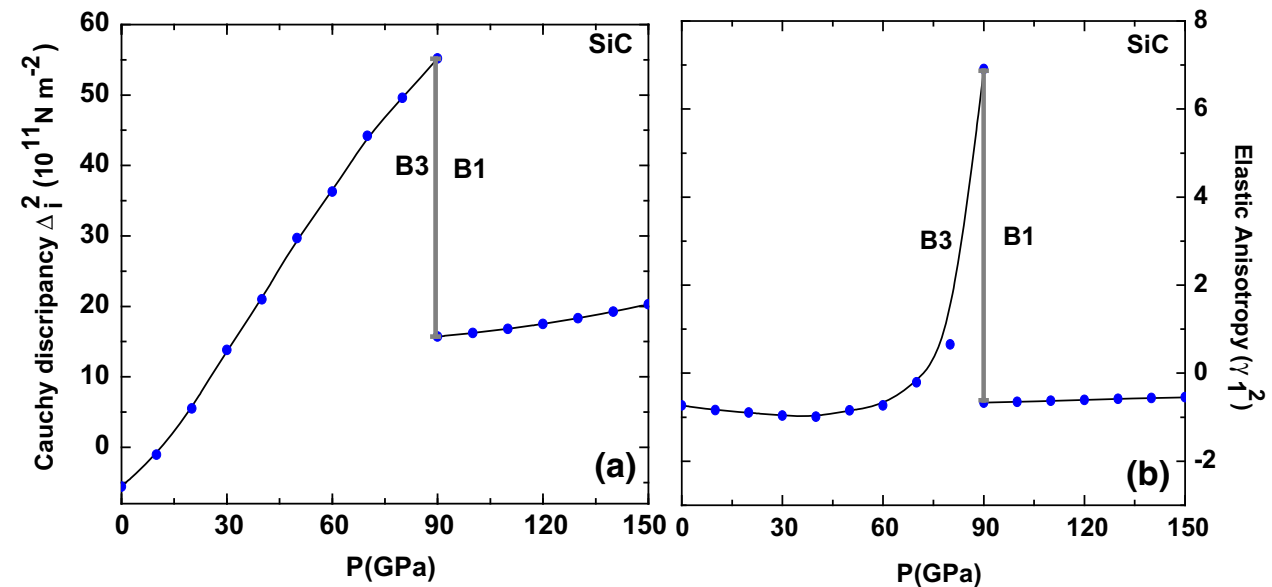

the crystal possesses the lowest potential energy among the mechanically stable lattices [66].

The elasticity in $3 C \mathrm{SiC}$ is thus probed by a non-central many body force potential which assumes that the interatomic forces have a certain shape and directionality. The Cauchy discrepancy is defined as $\Delta_{1}^{2}=C_{12}-C_{44}-2 P$. Here, $\Delta_{1}^{2}$ is a measure of the contribution from the noncentral many body force. However, for pure central interatomic potentials, Cauchy relation is $C_{12}=C_{44}+2 P$.

At zero pressure, the Cauchy discrepancy $\left(\Delta_{1}^{2}\right)$ in $\mathrm{SiC}$ is about $-5.588 \times 10^{10} \mathrm{Nm}^{-2}$. The $\Delta_{1}^{2}$ further enhances on increasing the pressure in both phases as depicted in Fig. 3a. In $\mathrm{SiC}$, larger deviation of $\Delta_{1}^{2}$ essentially points to the importance of the many body non-central (charge transfer and covalency) interaction in the interatomic potential and anharmonic effects are substantial at high pressures. The strength of non-central many body forces incorporating charge transfer interactions and covalency effects is witnessed by significant deviation in $\Delta_{1}^{2}$ at different pressures not only in ZB but also in RS structure, although weak. The importance of many body non-central forces and anharmonic effects is further explored by analysing the higher order elastic constants explicitly the thirdorder elastic constants. Usually, the anharmonic effects are noticeable at high pressure as reflected from elastic constants behaviour.

The anisotropy in second-order elastic constants is reflected from geophysical activities of various materials and alloys. The anisotropic parameter $\gamma$ is unity for isotropic elasticity. As far as cubic crystal is concerned, 
although it is isotropic in structure, it has elastic anisotropy other than unity. This is a consequence of fourth rank tensor property of elasticity.

We define elastic anisotropic parameter $\gamma_{1}^{2}$ in terms of aggregate $C_{i j}$ as [67]:

$\gamma_{1}^{2}=\frac{C_{11}-C_{12}-2 C_{44}}{2 C_{44}}$

The pressure dependence of the elastic anisotropic parameter $\gamma_{1}^{2}$ in $\mathrm{SiC}$ is shown in Fig. 3b. It is evident that $\gamma_{1}^{2}$ in $\mathrm{SiC}$ is insensitive below transition pressure and also at low pressures. A jump has been noted at $P_{T}(=90 \mathrm{GPa})$ inferring first-order structural phase transition. Furthermore, $\gamma_{1}^{2}$ in $\mathrm{SiC}$ remains unaltered for $B 1$ phase for higher pressures. The value of anisotropic parameter $\gamma_{1}^{2}$ for $\mathrm{SiC}$ is given in Table 4 at $T=0 \mathrm{~K}$ and $P=0 \mathrm{GPa}$.

During mechanical processing, explicitly in fabrication the melting ranges of materials and alloys are substantial. The usage of an alloy in the applications as the success of the melting and casting operations depends on the correct selection of temperature. Once solidified and primary processed (rolling or forging), the melting temperature has little significance to designers, engineers and users. The melting temperature influences elevated temperature

Table 4 Calculated aggregate second-order elastic constants $\left(C_{11}\right.$, $C_{12}$ and $\left.C_{44}\right)$ and aggregate bulk modulus $\left(B_{T}\right)$, second-order elastic constant anisotropy parameter $\left(\gamma_{i}^{2}\right)$, isotropic shear modulus $\left(G_{H}\right)$, Voigt's shear modulus $\left(G_{\mathrm{V}}\right)$, Reuss's shear modulus $\left(G_{\mathrm{R}}\right)$, Young's modulus $(E)$, Poisson ratio $(v)$, First and second Lame constant $(\lambda, \mu)$, longitudinal $\left(v_{1}\right)$, shear $\left(v_{\mathrm{s}}\right)$ and average elastic wave velocity $\left(v_{\mathrm{m}}\right)$, Kleinman parameter $(\xi)$ and thermodynamical properties: Debye temperature $\left(\theta_{\mathrm{D}}\right)$ of silicon carbide in $B 3$ phase at temperature of about $300 \mathrm{~K}$

\begin{tabular}{ll}
\hline Materials parameter & $\mathrm{SiC}$ \\
\hline$C_{11}\left(10^{10} \mathrm{Nm}^{-2}\right)$ & $67.65,35.23[49]$ \\
$C_{12}\left(10^{10} \mathrm{Nm}^{-2}\right)$ & $8.31,14.04[49]$ \\
$C_{44}\left(10^{10} \mathrm{Nm}^{-2}\right)$ & $19.99,23.29[49]$ \\
$B_{T}\left(10^{10} \mathrm{Nm}^{-2}\right)$ & 28.1 \\
$C_{S}\left(10^{10} \mathrm{Nm}^{-2}\right)$ & 29.7 \\
$G_{H}\left(10^{10} \mathrm{Nm}^{-2}\right)$ & 23.43 \\
$G_{V}\left(10^{10} \mathrm{Nm}^{-2}\right)$ & 23.86 \\
$G_{R}\left(10^{10} \mathrm{Nm}^{-2}\right)$ & 22.99 \\
$E\left(10^{10} \mathrm{Nm}^{-2}\right)$ & $54.9,43.7[71]$ \\
$v$ & $0.174,0.167[71]$ \\
$\lambda\left(10^{10} \mathrm{Nm}^{-2}\right)$ & 12.47 \\
$\mu\left(10^{10} \mathrm{Nm}^{-2}\right)$ & 23.43 \\
$v_{\mathrm{l}}\left(\mathrm{ms}^{-1}\right)$ & $12,440,12,182[71]$ \\
$v_{\mathrm{s}}\left(\mathrm{ms}^{-1}\right)$ & $7819,7701[71]$ \\
$v_{\mathrm{m}}\left(\mathrm{ms}^{-1}\right)$ & 1824 \\
$\xi$ & 0.274 \\
$\theta_{\mathrm{D}}\left(\mathrm{K}^{-1}\right.$ & 708.60 \\
\hline
\end{tabular}

properties, such as creep strength, but the researchers have limited interest. The pressure dependence of the melting temperature: $T_{\mathrm{M}}=553 \mathrm{~K}+5.91 C_{11} \mathrm{~K} \mathrm{GPa}^{-1}$ for $\mathrm{SiC}$ in $B 3$ and $B 1$ phase is discerned in Fig. 4. It is noticed that $T_{\mathrm{M}}$ enhances with increased pressure or in other words the resistance to deformation by a stress increases. It is noticed that at zero pressure, the melting temperature of $\mathrm{SiC}$ is $2746 \mathrm{~K}$ consistent with reported value of about $3100 \pm 40 \mathrm{~K}[1,2]$. At $P_{T}(=90 \mathrm{GPa})$, its value is about $4000 \mathrm{~K}$ which enhances further in $B 1$ phase. An increase in $T_{\mathrm{M}}$ with variations in pressure infers the hardening or stiffening of the lattice. Higher melting temperature symbolizes higher shear modulus $(G)$, and Young's modulus $(E)$ values that we shall see later on. The data on its melting under high pressure are very limited and extremely contradictory, which does not allow one to make any conclusions about congruent or incongruent melting behaviour as well as the slope of the melting curve of $\mathrm{SiC}$.

$\mathrm{SiC}$ is the only compound in the $\mathrm{Si}(\mathrm{IV})-\mathrm{C}(\mathrm{IV})$ binary system and is obtained by electromelting high purity silica sand with petroleum coke, also of good quality. This melting takes place at high temperature about $2473 \mathrm{~K}$ and requires a large quantity of energy to produce. It also requires energy for it to dissociate into about $2 / 3 \mathrm{Si}$ and $1 / 3$ $\mathrm{C}$ in the induction furnace $[1,2]$. The better quality raw materials produce better quality $\mathrm{SiC}$, which is lower in nitrogen, sulphur, hydrogen and other trace elements. Figure 4 shows the temperature dependence of the melting temperature for $\mathrm{SiC}$ estimated from the $C_{11}$ elastic constant as discussed previously. At room temperature its value is about $4550 \mathrm{~K}$ which drops and is $4200 \mathrm{~K}$ at $1000 \mathrm{~K}$ in $B 3$ phase. The suppressed $T_{M}$ with increased temperature indicates that there is a decrease in the resistance to deformation by a stress induced due to temperature. The suppressed $T_{M}$ infers the weakening of the lattice as a result of thermal softening. Usually, SiC does not melt, it actually dissolves since its melting point is about $2973 \mathrm{~K}$. Its behaviour in the molten metal is similar to sugar dissolving in coffee. This aspect is very important for the use of SiC.

For cubic lattice, three second-order elastic constants and the six non-vanishing third-order elastic constants are obtained from crystal geometry. The anharmonicity of a crystal lattice is successfully probed in terms of higher order elastic constants. The third-order terms in the strain variables are deduced from derivatives of elastic energy (please see Appendix for both ZB B3 and RS B1 phases). For $\mathrm{SiC}$, the third-order aggregate elastic constants $C_{111}$, $C_{112}, C_{166}, C_{144}$, and $C_{456}$, are negative and only $C_{123}$ is positive at $P=0 \mathrm{GPa}$ i.e., in $B 3$ phase. We note that no such efforts have been made in the past for third-order elastic constants of SiC. Thus, deduced information on pressure-dependent $C_{i j k}$ will serve as a guide line for future 
Fig. 4 Variation of melting temperature $\left(T_{\mathrm{M}}\right)$ with pressure and temperature

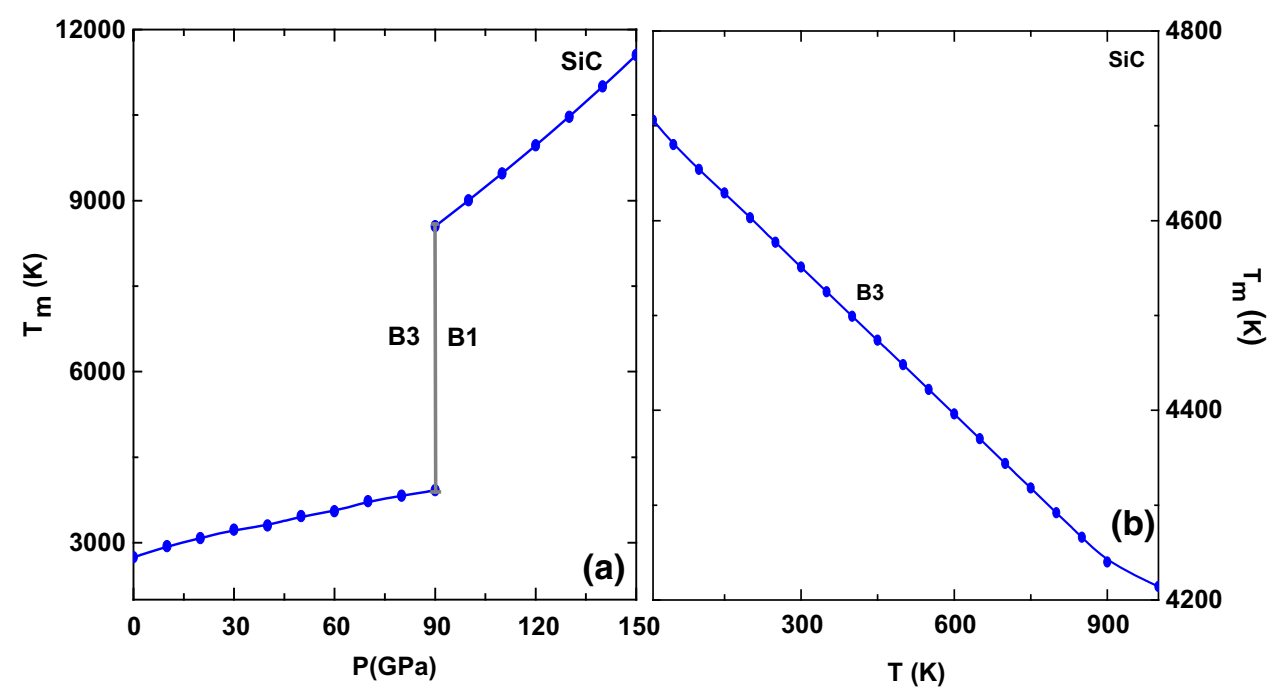

research and its application in materials technology. This information is useful as the structural strengths of a material are successfully known from the knowledge of elastic constants. The microstructures developed on the applied pressure and temperature can be known from thirdorder elastic constants variations.

The variation of aggregate third-order elastic constants (TOECs) with pressure for $\mathrm{SiC}$ in $B 3$ and $B 1$ phase is sketched in Fig. 5. For $\mathrm{SiC}$ in cubic phase, the $C_{144}$, and $C_{166}$ increases with enhancing pressure. Other TOECs as $C_{456}, C_{112}, C_{111}$, and $C_{123}$ infer a decreasing trend. We note that $C_{144}$ is remarkably larger as compared to other TOECs. Furthermore, aggregate elastic constants $C_{i j k}$ inferring the anharmonic effects are influenced by application of pressure in $\mathrm{SiC}$. In the present interatomic potential, $C_{i j k}$ are affected by the inclusion of secondnearest-neighbour interaction and are influenced by the short-range interactions (please see Eqs. 60-65 in the Appendix). Also, many body non-central forces as longrange Coulomb, charge transfer interactions and covalency are effective in $\mathrm{SiC}$. We comment that pressure-dependent $C_{i j k}$ behaviour can have a direct means to describe the interatomic forces at high pressure. It successfully cares the short-range forces, and a balance between long range and short-range forces.

As a next step, we intend to study the anisotropy among $C_{i j k}$. The equilibrium condition in the interatomic potential infers $B_{1}+B_{2}=-1.261 Z_{m}^{2}$. The Cauchy discrepancy $\Delta_{i}^{3}$ among third-order elastic constants are: (a) $\Delta_{1}^{3}=$ $C_{112}-C_{166}-2 P$; (b) $\Delta_{2}^{3}=C_{123}-C_{456}-2 P$; (c) $\Delta_{3}^{3}=$ $C_{144}-C_{456}-2 P$; and (d) $\Delta_{4}^{3}=C_{123}-C_{144}-2 P$. The $C_{i j k}$ at zero pressure i.e., in $B 3$ phase are influenced by contributions from both long-range and short-range interactions. Henceforth, $\Delta_{i}^{3}$ is an indicator of the contribution from the non-central many body force. Figure 5 a shows the variation of $\Delta_{i}^{3}$ as functions of pressure. The significant deviation in $\Delta_{i}^{3}$ is a natural consequence of the non-central many body forces as charge transfer interactions ions of $\mathrm{Si}$ and $\mathrm{C}$ atom and covalency effects caused by $\mathrm{Si}-\mathrm{Si}, \mathrm{Si}-\mathrm{C}$, and $\mathrm{C}-\mathrm{C}$ bonds apart from short-range interactions as the induced charge dipole-dipole and charge dipole-quadruple (van der Waals) interaction and the overlap repulsion. At zero pressure, $\Delta_{2}^{3}$ and $\Delta_{3}^{3}$ are positive, while to that $\Delta_{1}^{3}$ and $\Delta_{4}^{3}$ are negative in $B 3$ phase. A growth in $\Delta_{2}^{3}$ and $\Delta_{3}^{3}$ and decay in with enhanced pressure is witnessed with enhanced pressure till phase transition pressure. However, all $\Delta_{i}^{3}$ is negative in $B 1$ phase indicating the importance of non-central many body forces and anharmonic effects in $\mathrm{SiC}$ ceramics. As far as we know, there are no experimental results available for Cauchy discrepancy $\Delta_{i}^{3}$ in SiC.

For cubic crystal structures, the aggregate third-order elastic constants $C_{i j k}$ discern three anisotropy coefficients and three isotropic coefficients. It is useful to express linear combinations of the anisotropy coefficients and dividing them by the isotropic coefficients [68-70]. The anisotropy coefficients $\gamma_{i}^{3}$ are as follows:

$\gamma_{1}^{3}=\frac{3 C_{112}-C_{111}-12 C_{144}+12 C_{166}-16 C_{456}-2 C_{123}}{2 C_{123}}$

$\gamma_{2}^{3}=\frac{C_{111}-C_{123}-2 C_{144}}{2 C_{144}}$

$\gamma_{3}^{3}=\frac{C_{166}-C_{144}-2 C_{456}}{2 C_{456}}$

The pressure dependence of the elastic anisotropic parameter $\gamma_{i}^{3}$ for both phases is sketched in Fig. 5b. The elastic anisotropy $\left(\gamma_{2}^{3}\right.$ and $\left.\gamma_{3}^{3}\right)$ in $B 3$ phase is less sensitive. On the other hand, $\gamma_{1}^{3}$ shows variation with increase in pressure in $B 3$ phase. The anisotropy factor $\gamma_{1}^{3}$ is negative and shows a 
Fig. 5 Variation of aggregate third-order elastic constants $\left(C_{i j k}\right)$ with pressure, Cauchy discrepancy $\left(\Delta_{i}^{3}\right)$ and elastic anisotropy $\left(\gamma_{i}^{3}\right)$ in third-order elastic constant with pressure
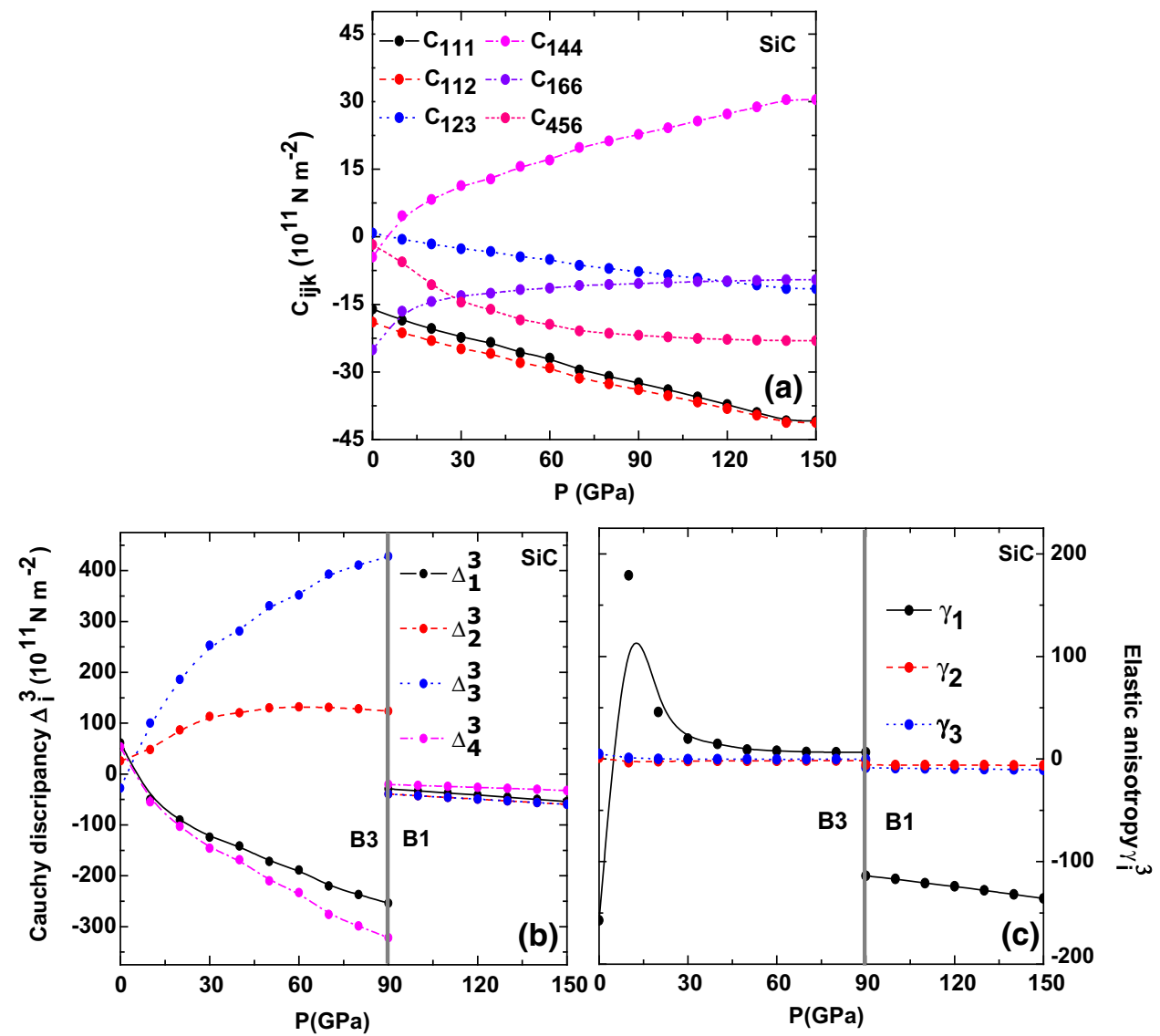

decreasing trend with pressure in $B 1$ phase at higher pressures. Values of $\gamma_{i}^{3}$ are given in Table 5 at zero temperature and pressure. As far as we know, there are no experimental and theoretical results available for comparison.

The strength and hardness are key issues for materials useful in technological applications. The mechanical strength and hardness of $\mathrm{SiC}$ can be known once elastic constants are known. We now determine elastic moduli at various pressures of $\mathrm{SiC}$ which are of substantial importance in engineering, geophysical and seismological application. The elastic properties are important in probing the bonding characteristic between adjacent atomic planes and the anisotropic character of the solid. Crystals leading to elasticity under application of pressure identify the response of a crystal under external strain. This valuable information is characterized by isotropic shear modulus $\left(G_{H}\right)$, and Young's modulus $(E)$. We note that hardness of polycrystalline materials is inhibited in elastic response as $G_{H}$ and $E$.

The resistance to volume change and resistance to reversible deformations upon applied pressures are accounted by Voigt-Reuss-Hill approximation using $G_{H}$ and $E$ following [68-70]:
$G_{H}=\frac{G_{\mathrm{V}}+G_{\mathrm{R}}}{2}$

Herein, $G_{\mathrm{V}}\left(G_{\mathrm{R}}\right)$ is Voigt's (Reuss's) shear modulus corresponding to the upper (lower) bound of $G_{H}$ values. The $G_{\mathrm{V}}\left(G_{\mathrm{R}}\right)$ is obtained from $C_{i j}$ assuming uniform strain throughout the sample as

$G_{\mathrm{V}}=\frac{C_{11}-C_{12}+3 C_{44}}{5}$

and

$\frac{5}{G_{\mathrm{R}}}=\frac{4}{\left(C_{11}-C_{12}\right)}+\frac{3}{C_{44}}$

Hardness of materials is usually measured in terms of isotropic shear modulus $\left(G_{H}\right)$, and Young's modulus $(E)$ or bulk modulus $\left(B_{T}\right)$. The bulk modulus infers resistance to volume change by applied pressure. The isotropic shear modulus $\left(G_{H}\right)$ measures the resistance to reversible deformations upon shear stress. In such a situation, $G_{H}$ is a better probe to measure hardness than the bulk modulus $\left(B_{T}\right)$. Table 4 illustrates the calculated isotropic shear modulus $\left(G_{H}\right)$, Voigt's shear modulus $\left(G_{\mathrm{V}}\right)$ and Reuss's shear modulus $\left(G_{\mathrm{R}}\right)$ for $\mathrm{SiC}$ ceramics at zero temperature and 
Table 5 Calculated aggregate third-order elastic constant $\left(C_{i j k}\right)$, third-order elastic constant anisotropy parameter $\left(\gamma_{i}^{3}\right)$, First and second Lame constant $(\lambda, \mu)$, longitudinal $\left(v_{1}\right)$, shear $\left(v_{\mathrm{s}}\right)$ and average elastic wave velocity $\left(v_{\mathrm{m}}\right)$, Kleinman parameter $(\xi)$ thermodynamical properties: force constant $(f)$, Reststrahlen frequency $\left(v_{o}\right)$, Debye temperature $\left(\theta_{\mathrm{D}}\right)$, and average elastic constant $(C)$, of Silicon Carbide in $B 3$ phase at zero pressure

\begin{tabular}{ll}
\hline Materials parameter & $\mathrm{SiC}$ \\
\hline$C_{111}\left(10^{10} \mathrm{Nm}^{-2}\right)$ & -160.70 \\
$C_{112}\left(10^{10} \mathrm{Nm}^{-2}\right)$ & -189.70 \\
$C_{123}\left(10^{10} \mathrm{Nm}^{-2}\right)$ & 8.33 \\
$C_{144}\left(10^{10} \mathrm{Nm}^{-2}\right)$ & -44.85 \\
$C_{166}\left(10^{10} \mathrm{Nm}^{-2}\right)$ & -250.90 \\
$C_{456}\left(10^{10} \mathrm{Nm}^{-2}\right)$ & -17.38 \\
$\gamma_{1}^{3}$ & -157.283 \\
$\gamma_{2}^{3}$ & 1.207 \\
$\gamma_{3}^{3}$ & 4.927 \\
$\lambda\left(10^{10} \mathrm{Nm}^{-2}\right)$ & 16.29 \\
$\mu\left(10^{10} \mathrm{Nm}^{-2}\right)$ & 16.47 \\
$v_{1}\left(\mathrm{~ms}^{-1}\right)$ & 12,400 \\
$v_{\mathrm{s}}\left(\mathrm{ms}^{-1}\right)$ & 7172 \\
$v_{\mathrm{m}}\left(\mathrm{ms}^{-1}\right)$ & 1688 \\
$\xi$ & 0.709 \\
$f\left(10^{5} \mathrm{dyne} \mathrm{cm}^{-1}\right)$ & 7.187 \\
$v_{0}\left(10^{12} \mathrm{~Hz}\right)$ & 6.867 \\
$\theta_{\mathrm{D}}(\mathrm{K})$ & 611.758 \\
$C_{\text {av. }}\left(10^{10} \mathrm{Nm}^{-2}\right)$ & 22.82 \\
\hline
\end{tabular}

pressure along with a comparison with the available theoretical results $[10,15,50,56,58,60]$.

A decreasing and then increasing nature of the isotropic shear modulus $G_{H}, G_{\mathrm{V}}$ and $G_{\mathrm{R}}$ is witnessed in $B 3$ phase of $\mathrm{SiC}$. On the other hand, a steep increase in $G_{H}, G_{\mathrm{V}}$ and $G_{\mathrm{R}}$ is seen with enhanced pressure in $B 1$ phase at higher pressures as plotted in Fig. 6a. The explanation of the above characteristics lies in a fact that both $G_{\mathrm{V}}$ and $G_{\mathrm{R}}$ are influenced by aggregate elastic constant $C_{44}$. Thus, $G_{V}$ decreases as $C_{44}$ decreases with enhanced pressure in $\mathrm{ZnS}$ phase. On the other hand, Reuss's shear modulus $\left(G_{\mathrm{R}}\right)$ pressure-dependent behaviour is integrated by combination of $C_{11}-C_{12}$ pressure-dependent behaviour as well as to that of $C_{44}$ pressuredependent behaviour. $G_{\mathrm{R}}$ initially decreases and then starts increasing at about $30 \mathrm{GPa}$ and is attributed to steep decrease in $C_{44}$. We end up by stating that above transition pressure an increase in $G_{H}, G_{\mathrm{V}}$ and $G_{\mathrm{R}}$ support our earlier prediction about mechanical stiffening of lattice.

The high temperature studies of materials at ambient pressure lead to the performance of a material in terms of (a) the understanding of vibrational anharmonicity that is associated with the relative interplay of long-range and short-range potential energy function, (b) thermal response in terms of softening or hardening and (c) the elastic behaviour of materials as the elasticity, extensibility, acoustic transmission velocity, Debye temperature, specific heat, and thermal conductivity.

The high temperature investigations cause laboratory difficulties and structural changes make the phenomenon more amenable to interpretation. Figure $6 \mathrm{~b}$ displays the $G_{H}, G_{\mathrm{V}}$ and $G_{\mathrm{R}}$ behaviour of $3 C \mathrm{SiC}$ ceramics as functions of temperature (at zero pressure). We note that the isotropic shear modulus of $\mathrm{SiC}$ is decreasing with enhanced temperature. The steep decrease of $G_{H}, G_{\mathrm{V}}$, and $G_{\mathrm{R}}$ is in accordance with aggregate second-order elastic constant $C_{i j}$ behaviour with temperature. Suppressed $G_{H}, G_{\mathrm{V}}$ and $G_{\mathrm{R}}$ as functions of temperature infer the weakening of the lattice as a result of thermal softening.

The tensile modulus as Young's modulus $(E)$ further elaborates the stiffness property. $E$ is also defined in terms of Reuss's shear modulus $\left(G_{H}\right)$, and bulk modulus $\left(B_{T}\right)$ :

$E=\frac{9 G_{\mathrm{H}} B_{T}}{G_{\mathrm{H}}+3 B_{T}}$

The tensile strength as Young's modulus $(E)$ for $3 C \mathrm{SiC}$ is illustrated in Table 4 for $\mathrm{ZnS}$ (B3) phase along with a comparison with the reported data $[10,15,50,55,56,60$, $62,64,65]$. The model calculations presented here lead to $E$ value of about $411 \mathrm{GPa}$ at zero pressure. It is known that the material is stiffer if its Young's modulus is high [ $E$ of steel, graphene and diamond is $\sim 200,1000$ and $1220 \mathrm{GPa}$. As inferred $\mathrm{SiC}$ is less stiff as compare to graphene and diamond. The pressure dependence of the Young's modulus $(E)$, of $\mathrm{SiC}$ is sketched in Fig. 7a. A decreasing trend of $E$ in $B 3$ phase infers the weakening of tensile strength till $90 \mathrm{GPa}$ and an increasing trend of $E$ in $B 1$ phase essentially identifies more stiffening. Looking to the wide usage of $\mathrm{SiC}$ in gas turbines, heat exchangers, ceramics fans, radar, microwave, solar cell, and highvoltage devices, these properties are worth investigating.

Figure $7 \mathrm{~b}$ illustrates the temperature dependence of the tensile strength as Young's modulus $(E)$ for $3 C \mathrm{SiC}$ ceramics. A decreasing trend is inferred with increase in temperature. Matsumoto and researchers have reported the Young's modulus and Poisson's ratio of $\mathrm{SiC}$ ceramics at temperatures $>1400{ }^{\circ} \mathrm{C}$ using laser ultrasonics coupled with Fabry-Pérot interferometry as well ultrasonic pulse method [71]. It is reported that $E$ is about $438 \mathrm{GPa}$ at $T=273 \mathrm{~K}$ and shows a decreasing behaviour with increasing temperature. The model calculations presented here lead to a value of about $549 \mathrm{GPa}$ at room temperature which is comparable to reported $E$ of $437 \mathrm{GPa}$ for $3 C \mathrm{SiC}$ ceramics [71]. A reduction of $E$ with temperature is a signature of bond weakening or thermal softening.

We now make a comparison of tensile strength $E(P)$ and $E(T)$ of $3 C \mathrm{SiC}$ ceramics. It is noted that 
Fig. 6 Variation of Isotropic shear modulus $\left(G_{H}, G_{\mathrm{V}}\right.$, and $\left.G_{\mathrm{R}}\right)$ with pressure and temperature
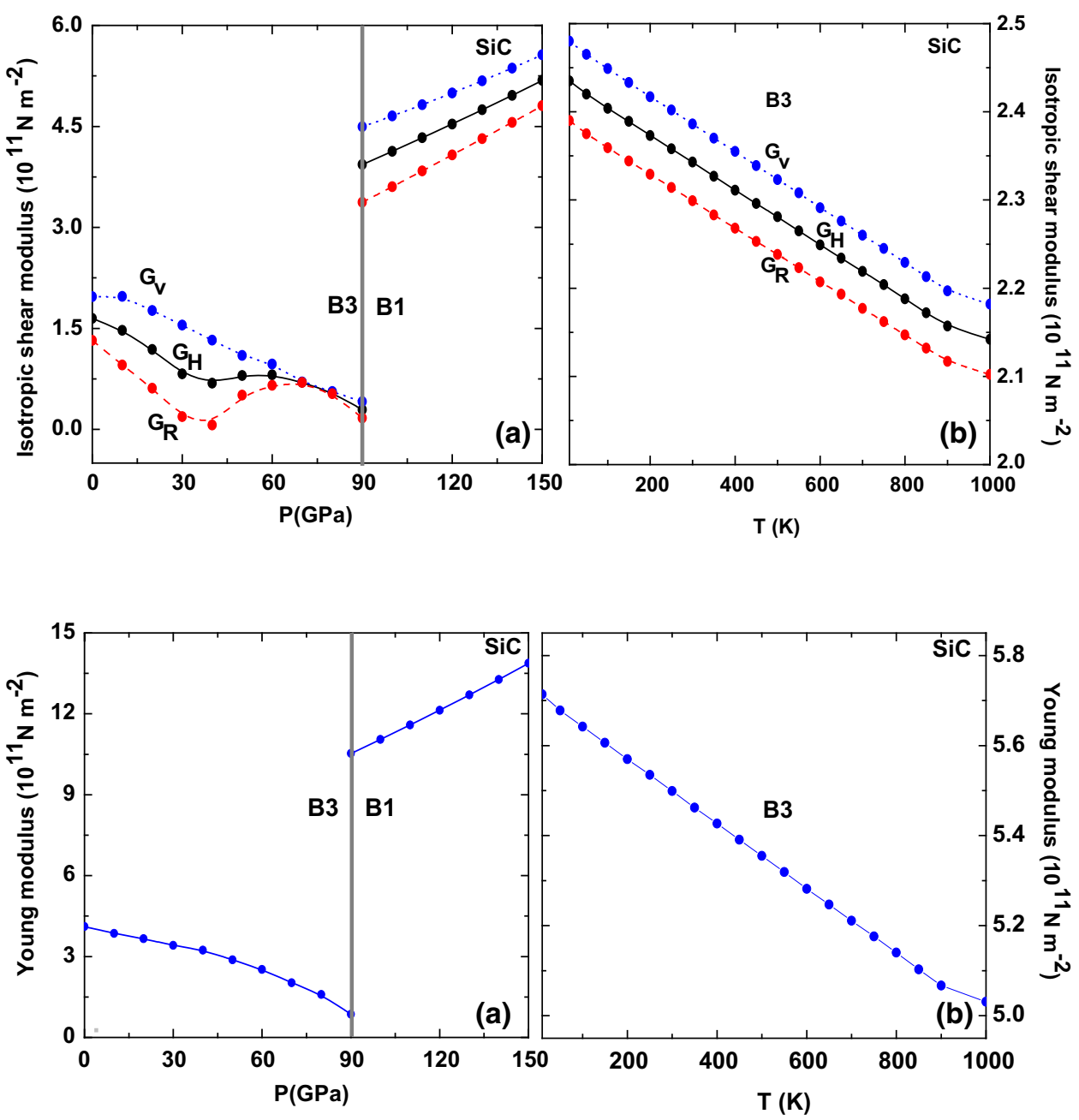

Fig. 7 Variation of Young's modulus $(E)$ with pressure and temperature
$E(P)$ values are smaller than $E(T)$ for $\mathrm{SiC}$ at low pressures and temperatures. It implies that $E(P)$ and $E(T)$ are although susceptible to external variables as pressure and temperature, but it is their $\mathrm{Si}$ and $\mathrm{C}$ ions in $3 \mathrm{C} \mathrm{SiC}$ ceramics that makes $\mathrm{SiC}$ lattice to be mechanical hard due to bond strengthening and thermal soft due to bond weakening. It is worth commenting that while tailoring the composites based on $\mathrm{SiC}$, one should seek its performance in terms of tensile strength.

Another interest in $3 C \mathrm{SiC}$ is to probe the ductile and brittle nature of Silicon and Carbon-based alloys are important and predicted from the knowledge of secondorder elastic constants. Ductile materials as Steel and aluminium sustain large strains before rupture, while to that brittle materials as glass and cast iron fractured at lower strains. For materials design and advances in metallurgy as well composite technology, the materials response for applied pressures and temperatures are often gauzed by stress-strain characteristics. Composites are predictive as depending on the external variable (pressure and temperature), the response of the constituent element is tailored in terms of ductile or brittle nature. Also ductile element in composites may become brittle as the pressure or temperature is increased or decreased. Once the pressure dependence of shear modulus $\left(G_{H}\right)$, and Young's modulus $(E)$ or bulk modulus $\left(B_{T}\right)$ is known, it is worth investigating ductile and brittle nature of $\mathrm{SiC}$.

The shear modulus $\left(G_{H},\right)$ measures the resistance to plastic deformation, while the bulk modulus $\left(B_{T}\right)$ probes the resistance to fracture. An empirical relation in terms of the ratio of these moduli is known to differentiate ductile and brittle nature. In accordance with the Pugh's ratio $\phi\left(=B_{T} /\right.$ $\left.G_{H}\right)>1.75$, the material sustains large strains before rupture i.e., the ductile response. On the other hand, for $\phi \leq 1.75$ the material is brittle. Empirically, 1.75 is a critical value that separates ductile and brittle response of materials [72].

In Fig. 8a, we have shown the Pugh ratio $\phi$ as functions of pressure. Note that $\mathrm{SiC}$ is ductile in $\mathrm{ZnS}$ phase (at zero as well at low pressures). Brittle nature is noticed at higher pressures in $\mathrm{ZnS}$ phase. Furthermore, brittle response of $\mathrm{SiC}$ is also noticed in rock salt structure. Hence, ceramics $\mathrm{SiC}$ is ductile/brittle irrespective of any structural 
transformations. To our knowledge, perovskite are ductile/ brittle that is a test for its deformation before fracture. In materials performance ductility is a powerful probe for quality control. It provides a means to assess the level of impurities and proper processing of a material. Hence, we classify $3 C \mathrm{SiC}$ as brittle material at higher pressures and ductile at zero pressure on the basis of Pugh ratio. The magnitude of elastic constants and shear moduli agrees with ductility degree of $\mathrm{SiC}$ in both $B 3$ and $B 1$ phase.

The Poisson's ratio is another measure to differentiate the ductility and brittleness of materials [73]. The critical value of Poisson's ratio (in terms of $B_{T}$ and $G_{H}$ ) $v$ is 0.33 that separates ductile and brittle nature of any material. If $v>0.33$, the material is ductile, and for $v<0.33$, the brittle response of material is observed such as ceramics. However, Poisson's ratio lies in between -1.0 and 0.5 which are the lower and upper bounds. The lower bound is a signature of the response of the materials that do not change its shape and the upper bound indicates that the volume is unchanged. The Poisson's ratio $v$ in terms of bulk modulus $B_{T}$ and the shear modulus $G_{H}$ is expressed as [68-70],

$v=\frac{1}{2}\left[3 \frac{B_{T}}{G_{\mathrm{H}}}-2\right]\left[3 \frac{B_{T}}{G_{\mathrm{H}}}+1\right]^{-1}$

Figure 8a summarizes the pressure-dependent results of Poisson's ratio $v$. $\mathrm{SiC}$ in $\mathrm{ZnS}$ phase documents mixed behaviour. At zero pressure ( $P=0 \mathrm{GPa})$, the value of $v$ is about 0.25 for $\mathrm{SiC}$. At lower pressures, $\mathrm{SiC}$ is brittle, above $15 \mathrm{GPa}$ and till phase transition pressure, ductile nature of $\mathrm{SiC}$ is observed. Above $P_{T}(=90 \mathrm{GPa})$, the value of $v$ is about 0.34 for $B 1$ phase. Above $P_{T}$, at higher pressures, in the RS structure, $\mathrm{SiC}$ remains brittle with increasing pressure. Deduced value of $v$ is in good agreement with available experimental and theoretical results $[5,10,11$, $15,47,50,56,59,63]$. As per definition of Poisson's ratio, $3 C \mathrm{SiC}$ is brittle. It should be pointed out here that both Pugh ( $\mathrm{SiC}$ as ductile) and Poisson's ratio ( $\mathrm{SiC}$ as brittle) give contradictory results. The two empirical rules only differ on the exact border between the two types of behaviour. In view of this, the pressure-dependent variations of $\mathrm{SiC}$ allow it to consider as a borderline case between the classes of ductile and brittle materials. We note the ceramics are brittle, but perovskites are ductile.

The Poisson's ratio $v$ behaviour as a function of temperature is sketched in Fig. 8b. The Poisson's ratio $v$ is independent of temperature and is consistent with the earlier measured $v$ by laser ultrasonics method [71]. Beginning from zero temperature and at high temperatures in $\mathrm{ZB}$ phase, $v \approx 0.174$ is obtained for $3 C \mathrm{SiC}$. The temperature-dependent Poisson's ratio reflects brittle nature of $\mathrm{SiC}$, while to that a borderline case between the classes of ductile and brittle materials is known from pressure dependence. Poisson's ratio is thus an effective indicator to control the level of impurities and processing of $\mathrm{SiC}$ ceramics. For covalent materials, $v$ is small $(v \sim 0.1)$, whereas for metallic materials $v$ is typically 0.33 . It is worth mentioning that agreement with experimental and theoretical data is not fortuitous, but it is attributed to proper parametrization and formulation of potential with non-central many body forces as charge transfer interactions ions of $\mathrm{Si}$ and $\mathrm{C}$ atom and covalency effects caused by $\mathrm{Si}-\mathrm{Si}, \mathrm{Si}-\mathrm{C}$, and $\mathrm{C}-\mathrm{C}$ bonds apart from short-range interactions as the induced charge dipoledipole and charge dipole-quadruple (van der Waals) interaction and the overlap repulsion.

Usually, the elastic moduli describe only reversible response of a material to small strain near equilibrium. The intrinsic strength of a material reflects permanent plastic deformation at large shear strain. Consequently, to further understand the behaviour of $\mathrm{SiC}$ in terms of Vickers hardness: $H_{V}=2 \quad\left(\phi^{2} G_{H}\right)^{0.585}-3$. Here, $\phi=B_{T} / G_{H}$. Figure 9a illustrates the theoretical Vickers hardness as a function of pressure. It is clear from the plot that the Vickers hardness $H_{V}$ decreases in $B 3$ phase and then increases in $B 1$ phase with increase in pressure, which
Fig. 8 Variation of Poisson's ratio $(v)$ and Pugh's modulus ratio $(\Phi)$ with pressure and temperature
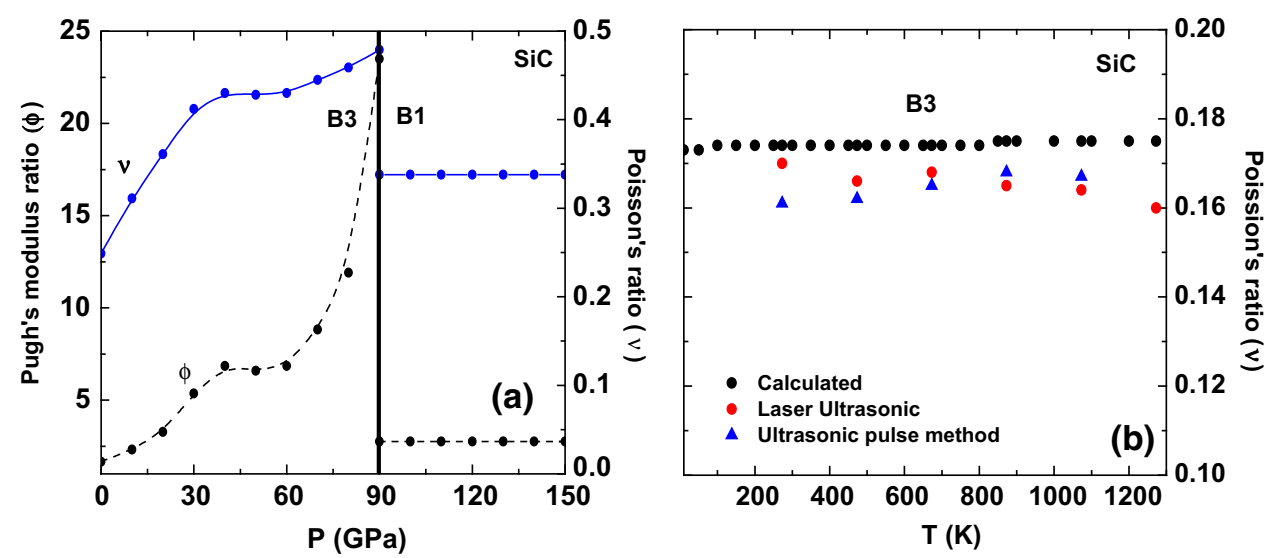
indicates that $\mathrm{SiC}$ becomes hard under pressure implying its good mechanical properties. Figure 9b shows Vickers hardness as a function of temperature for $\mathrm{SiC}$. It is clear from the plot that the Vickers hardness $H_{V}$ decreases in $B 1$ phase, which indicates that $\mathrm{SiC}$ gets softened with enhanced temperature. Apart from the elastic anisotropy of crystals, the hardness is important to discuss their properties because it is highly correlated with the possibility of inducing microcracks in materials.

SiCs are promising materials with wide range of applicability's with effective mechanical properties. Usually, materials elastic properties are a source of valuable information where materials mechanics is significant as the knowledge of deformational characteristics of materials is essential in engineering design and construction of effective structures. Having, understood the materials elastic behaviour using Bulk modulus $\left(B_{T}\right)$, shear modulus $\left(G_{H}\right.$, $G_{\mathrm{V}}$ and $G_{\mathrm{R}}$ ), and Young modulus $(E)$, in the following, we will use our calculated elastic constants to discuss the compressional and shear wave velocity in ceramics $\mathrm{SiC}$. The velocities of the longitudinal $v_{1}$ and shear waves $v_{\mathrm{S}}$ are known from the Lamé's constants, $\lambda$ and $\mu$. The compressional wave with velocity $v_{1}$ propagates back and forth in a crystal. The shear waves with velocity $v_{\mathrm{s}}$ go up and down.

The Lamé's constants are related to Young's modulus $(E)$ and Poisson's ratio (v) [correlating the bulk modulus $B_{T}$ and the shear modulus $\left.G_{H}\right]$. The first Lamé's constant $(\lambda)$ measures the compressibility of the material. The second Lamé's constant $(\mu)$ infers its shear stiffness [68-70]. The Lamé's constants $(\lambda$ and $\mu)$ are expressed as:

$\lambda=\frac{v E}{(1+v)(1-2 v)}$

$\mu=\frac{E}{2(1+v)}$
Figure 10a displays the pressure dependence of the first and second Lamé's constants $(\lambda, \mu)$. Starting from zero pressure and at high pressures, both Lamé's constants $(\lambda, \mu)$ are positive. Note that the Lamé's constant $(\lambda)$ can be negative; however, for most materials it is also positive. The second Lamé's constant $(\mu)$ is positive. An increasing trend of compressibility $(\lambda)$ of $\mathrm{SiC}$ is witnessed in terms of $\lambda$ as seen in both ZB and RS structures and is attributed to mechanical hardening of lattice. The decreasing trend in $B 3$ phase and increasing trend in $B 1$ phase of shear modulus $\left(G_{H}, G_{\mathrm{V}}\right.$ and $\left.G_{\mathrm{R}}\right)$ results in a decreasing behaviour of shear stiffness $(\mu)$ in $B 3$ phase and then enhanced shear stiffness in $B 1$ phase. Note that $\lambda$ and $\mu$ together constitute a parameterization of the elastic moduli for homogeneous isotropic media. The values of pressure-dependent Lamé's constants $(\lambda, \mu)$ are documented in Table 5. Deduced values of Lamé's constants $(\lambda, \mu)$ could not be compared due to lack of data on SiC. Figure 10b summarizes the temperature-dependent behaviour of Lamé's constants $(\lambda, \mu)$, for $3 C \mathrm{SiC}$. It is noticed that both Lamé's constants $(\lambda, \mu)$ are decreasing with increasing temperature. Thus, both compressibility and shear stiffness showed decreasing trend with temperature variations and identifies once again the thermal softening of SiC.

The Lamé's constants $(\lambda, \mu)$ determine the longitudinal (shear) wave velocity as

$v_{1}=\left[\frac{\lambda+2 \mu}{\rho}\right]^{\frac{1}{2}}$

$v_{\mathrm{s}}=\left[\frac{\mu}{\rho}\right]^{\frac{1}{2}}$

Here, $\rho$ is the density. The average wave velocity $v_{\mathrm{m}}$ has been approximately given by

$v_{\mathrm{m}}=\left[\frac{1}{3}\left(\frac{2}{v_{t}^{3}}+\frac{1}{v_{s}^{3}}\right)\right]^{-\frac{1}{3}}$
Fig. 9 Variation of Hardness $\left(H_{V}\right)$ as a function of pressure and temperature
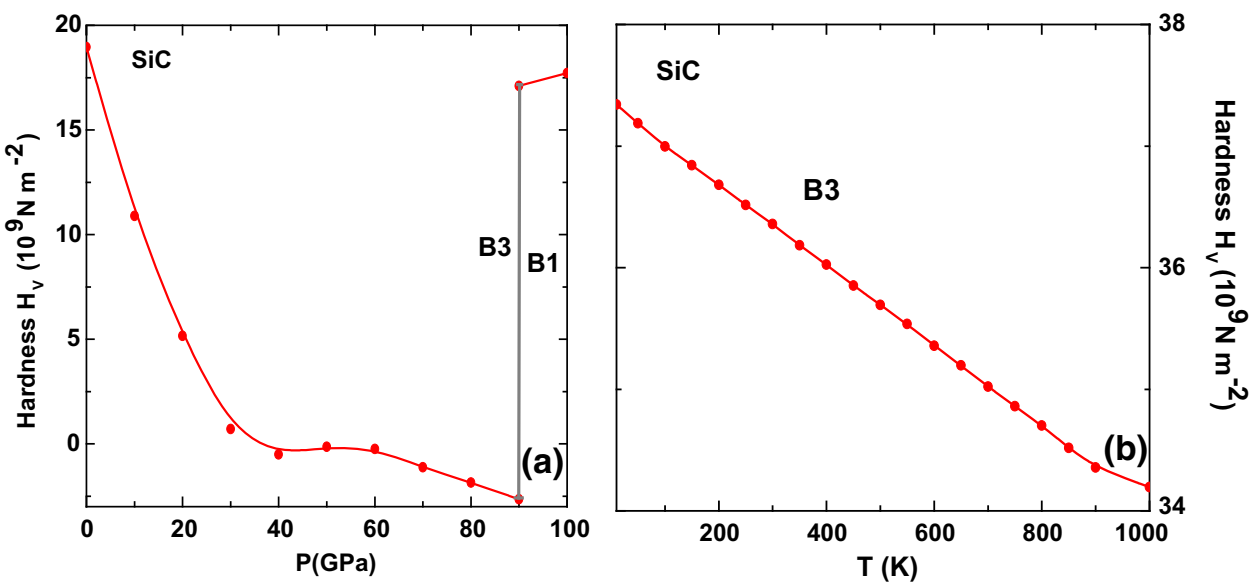
The compressional longitudinal (shear) wave velocity thus depends on density of the material as well on Bulk modulus $\left(B_{T}\right)$, shear modulus $\left(G_{H}, G_{\mathrm{V}}\right.$ and $\left.G_{\mathrm{R}}\right)$, and Young modulus $(E)$. We note that Bulk modulus $\left(B_{T}\right)$ has a strong dependence on the density of the material, Young modulus $(E)$ displays a weak dependence, while the shear modulus $\left(G_{H}\right)$ is independent of density.

Figure 11a displays the pressure dependence of the longitudinal (shear) wave velocity in SiC. In $\mathrm{ZB}$ phase, beginning from zero pressures and at high pressures, compressional wave velocity $v_{1}$ increases while to that shear wave velocity $v_{\mathrm{s}}$ decreases. Both compression and shear waves increase with enhanced pressure in $B 1$ structure. Deduced values of longitudinal, shear and average elastic wave velocities propagating in $\mathrm{SiC}$ are illustrated in Table 5 at zero temperature and pressure. Figure $11 \mathrm{~b}$ represents the temperature dependence of the longitudinal (shear) velocity, respectively. It is noticed that both longitudinal (shear) wave velocity decreases in $B 3$ phase with enhanced temperature. The values of the longitudinal, shear and average elastic wave velocities propagating in $3 C \mathrm{SiC}$ ceramics are documented in Table 4 at room temperature. Deduced values of wave velocities are in good agreement with the measured values by laser ultrasonics method [71]. The high temperature behaviour of longitudinal (shear) wave velocity for $\mathrm{SiC}$ can be considered as predictive studies as they cannot be compared due to unavailability of high temperature data.

The Navier's equation is also used to determine the longitudinal and the shear wave velocity [74, 75]. These are written in terms of Reuss's shear modulus $\left(G_{H}\right)$, and bulk modulus $\left(B_{T}\right)$ as

$v_{l}=\left[\frac{3 B_{T}+4 G_{H}}{3 \rho}\right]^{\frac{1}{2}}$

$v_{s}=\left[\frac{G_{H}}{\rho}\right]^{\frac{1}{2}}$

Elastic, plastic and molten state properties with pressure as controlling variable are useful for tailoring composites. The Lamé's constants $(\lambda, \mu)$ are of substantial interest for plastic materials. Referring to equations 32 and 33, we note that as the Poisson's ratio ( $v)$ increases, the Lamé's constants $(\lambda, \mu)$ numerically approach the bulk modulus $(E)$. For fluids, the Reuss's shear modulus $\left(G_{H}\right)$ vanishes as the viscosity of the fluid approaches zero. The above is
Fig. 10 Variation of Lamé's constant $(\lambda, \mu)$ with pressure and temperature

Fig. 11 Variation of elastic wave velocity $v_{1}$ and $v_{\mathrm{s}}$ with pressure and temperature
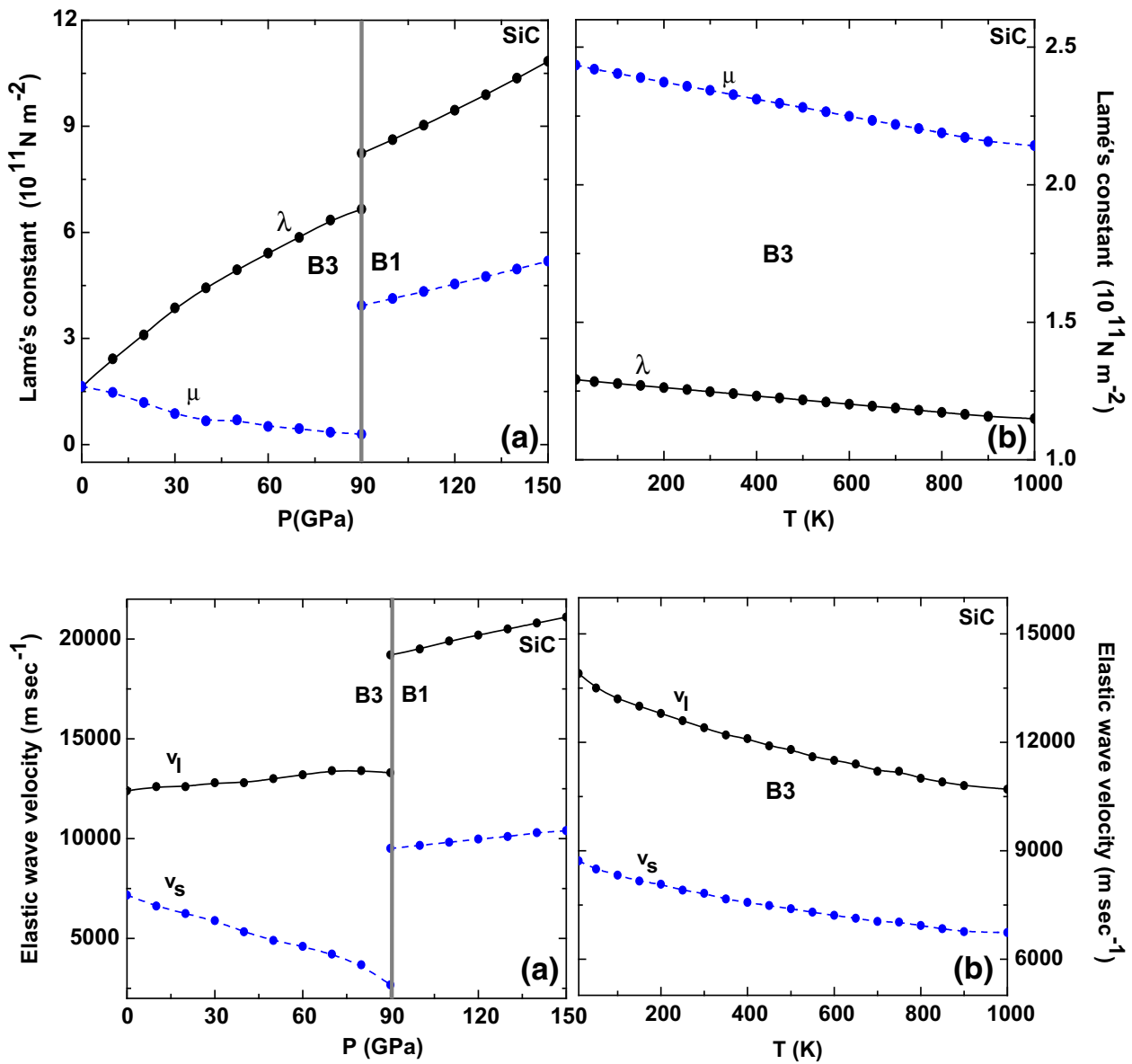
important in context of the present computation and can also be cross-checked from the relation between bulk modulus and Reuss's shear modulus: $B_{T}=\lambda+2 G_{H} / 3$. Furthermore, $G_{H}$ approaches zero for fluids and hence the Poisson's ratio is $\sim 0.5$.

The response to deformations against bond bending or bond-angle distortion is relevant for materials with promising technological applications and also a test to validate the many body non-central potential that we dealt with. The aggregate elastic constants infer the elastic properties of material that undergo stress, deform and then recover after returns to its original shape after stress ceases. The nature of elastic constants in solids holds a great importance to elucidate the microscopic nature interatomic bonding, equations of state, and vibrational density of states. The above can be understood in terms of Kleinman parameter, $\xi$, which describes the relative positions of the cation and anion sub-lattices under volume-conserving strain distortions for which positions are not fixed by symmetry [76, 77]. We have explored its applicability to ceramics $\mathrm{SiC}$ using [78].

$\xi=\frac{C_{11}+8 C_{12}}{7 C_{11}+2 C_{12}}$

Deduced value of $\xi \approx 0.709$ is documented in Table 5 for $3 C \mathrm{SiC}$ at zero temperature and pressure. The value of $\xi \approx 0.274$ is obtained at room temperature and is reported in Table 4 for $3 C$ SiC. A low value of $\xi$ implies a large resistance against bond bending or bond-angle distortion and vice versa [79]. Thus, $\mathrm{SiC}$ shows resistance against bond bending or bond-angle distortion and hence is of immense use in heat exchangers and ceramics fans. As far as we know, there is no experimental result available for Kleinman parameter and is considered as a prediction study. For both ZB and RS structures, the knowledge of elastic constants at variable pressure is worth investigating aimed for practical applications related to the mechanical properties of a solid: load deflection, thermo elastic stress, internal strain, elastic wave velocities, and fracture toughness.

Physical properties as thermal expansion, heat capacity and Grüneisen parameter can be explained with higher order terms of the interaction potential. The thermal process in terms of heat conduction is hindered if one considers a solid to be perfectly harmonic and thermal conductivity will be infinitely large. Thus, the role of anharmonic effects or phonon decay is important as enhanced pressures as well temperatures allows a change in volume or dimensions. The pressure-dependent calculations of elastic constants will provide a measure of the accuracy of the calculation of forces in $\mathrm{SiC}$ as well the mechanical stiffening or hardening attributed to $\mathrm{Si}-\mathrm{Si}$,
$\mathrm{C}-\mathrm{C}$, and $\mathrm{Si}-\mathrm{C}$ bond compression and bond strengthening due to lattice vibration.

We express the molecular force in the absence of the Lorentz effective field [19-26].

$f=\frac{1}{3}\left[\frac{\mathrm{d}^{2}}{\mathrm{~d} r^{2}} U_{S R}(r)-\frac{2}{r_{0}} \frac{\mathrm{d}}{\mathrm{d} r} U_{S R}(r)\right]_{r=r_{0}}$,

The force constant is a function of the second-order derivatives of the short-range (SR) overlap repulsive potential and as well the charge dipole-dipole and charge dipole-quadruple van der Waals potential. The Reststrahlen frequency is obtained from the force constant using

$v_{0}=\frac{1}{2 \pi}\left[\frac{f}{\mu}\right]^{1 / 2}$,

Here, $\mu$ is the reduced mass of $\mathrm{SiC}$.

The Grüneisen parameter $\gamma_{\mathrm{G}}$ is a ratio of second and first-order derivatives of the potentials enable us to discuss the anharmonic effects in a crystal. We express $\gamma_{\mathrm{G}}$ as

$\gamma_{\mathrm{G}}=-\frac{r_{0}}{6}\left[\frac{U^{\prime \prime \prime}\left(r_{0}\right)}{U^{\prime \prime}\left(r_{0}\right)}\right]$

The pressure dependence of Grüneisen parameter is sketched in Fig. 12a for $3 C \mathrm{SiC}$ in $\mathrm{ZnS}$ (B3) and $\mathrm{RS}$ (B1) phases. A linear decrease of $\gamma_{\mathrm{G}}$ with pressure in both phases is observed. The Grüneisen constant jump $\left[\gamma_{\mathrm{G}}\right.$ $(B 3-B 1)]$ at $P_{T}$ is about $7 \%$ in $3 C \mathrm{SiC}$ ceramics. Deduced $\gamma_{\mathrm{G}}$ value of about 1.015 for $3 C \mathrm{SiC}$ in $\mathrm{ZnS}$ (B3) phase is in good agreement with available theoretical results $[5,11,61]$. On the other hand, Fig. $12 \mathrm{~b}$ discerns temperature dependence of Grüneisen parameter with a value of about 0.98 at $1000 \mathrm{~K}$. For most of the solids, Grüneisen parameter ranges from 1.5 to 2.5. As Grüneisen constant $\gamma_{\mathrm{G}}$ behaviour is influenced by ratio of second and first-order derivatives of the potentials, we may thus comment that anharmonicity is significant in SiC ceramics. Note that these results are further validated by inelastic neutron scattering measurements to probe phonon frequencies as a function of the crystal volume.

The isothermal compressibility $(\beta)$ is known from second-order derivative of the potential as

$\beta=\left[\frac{r_{0}^{2}}{9 V}\left\{U^{\prime \prime}\left(r_{0}\right)\right\}\right]^{-1}$

The isothermal compressibility $(\beta)$ is another interesting thermodynamical property which invokes the bond compression or strengthening on application of pressure. With this motivation, we have computed and sketched isothermal compressibility $(\beta)$ with pressure in Fig. 13a. For SiC, at zero pressure, $\beta$ is about 0.024 which is comparable with available theoretical result of $0.1518[5,11]$. Note that the 
lattice of $\mathrm{SiC}$ is stiffened with increased pressure in $B 3$ phase. At phase transition pressure it gets softened. Away from phase transition pressure and at higher pressures i.e., in $B 1$ phase $\beta$ again becomes stiff. The mechanical stiffening is reflected from all elastic response of $\mathrm{SiC}$. It is attributed to $\mathrm{Si}-\mathrm{Si}, \mathrm{C}-\mathrm{C}$, and $\mathrm{Si}-\mathrm{C}$ bond compression and bond strengthening due to lattice vibration in both $B 3$ and $B 1$ phase. We comment that the compressibility $(\beta)$ at zero pressure is reduced by about $1 \%$ of that at transition pressure $P_{T}$ in $B 3$ phase in SiC. However, the magnitude of $\beta$ at transition pressure $P_{T}$ is about $0.3 \%$ of that at higher pressures $(\sim 150 \mathrm{GPa})$. We identify that $\mathrm{SiC}$ documents giant lattice softening at the boundary of $B 3-B 1$ phase transition.

The compressibility is useful in engineering applications as it measures the performance of a material explicitly the elasticity, extensibility, and thermal conduction. The isothermal compressibility $(\beta)$ as functions of temperature is shown in Fig. 13b. It is seen that $\beta$ displays a steep increase in $B 3$ phase. The compressibility is thus tunable with applied pressure and temperature stimuli and plays a vital role in tailoring materials and composites.
We further compute Debye temperature $\left(\theta_{\mathrm{D}}\right)$ to shed further light on mechanical stiffened and thermal softened characteristics of $\mathrm{SiC}$ following [80-84]:

$$
\theta_{\mathrm{D}}^{3}=\frac{3.15}{8 \pi}\left(\frac{h}{k_{\mathrm{B}}}\right)^{3}\left(\frac{r}{M}\right)^{\frac{3}{2}}\left(\frac{C_{11}-C_{12}}{2}\right)^{\frac{1}{2}}\left(\frac{C_{11}+C_{12}+2 C_{44}}{2}\right)^{\frac{1}{2}} C_{44}^{\frac{1}{2}},
$$

where, $M$ is the acoustic mass of SiC. The notations $h$ and $k_{\mathrm{B}}$ are the Planck and Boltzmann constants.

The Debye temperature as functions of pressure is plotted in Fig. 14a for SiC. At zero pressure, $\theta_{\mathrm{D}}$ is about $611 \mathrm{~K}$. It is noticed that with enhanced pressure, $\theta_{\mathrm{D}}$ decreases in $B 3$ phase for $\mathrm{SiC}$. The Debye temperature from $B 3$ to $B 1$ phase is jumped by $655 \mathrm{~K}$ at $P_{T}$. On the other hand, $\theta_{\mathrm{D}}$ showed an increase in $B 1$ phase. Deduced value of $\theta_{\mathrm{D}}$ is listed in Table 5. Suppressed $\theta_{\mathrm{D}}$ in $B 1$ phase at higher pressure indicates the mechanical stiffening of lattice and giant softening at $P_{\mathrm{T}}$ and above pressures.

Debye temperature is a function of temperature and its value varies from technique to technique as well as depends on the sample quality. Usually, a standard deviation in $\theta_{\mathrm{D}}$ of about $15 \mathrm{~K}$ is agreeable. The change in the
Fig. 12 Varaition of Gruneisen parameter $\left(\gamma_{\mathrm{G}}\right)$ with pressure and temperature

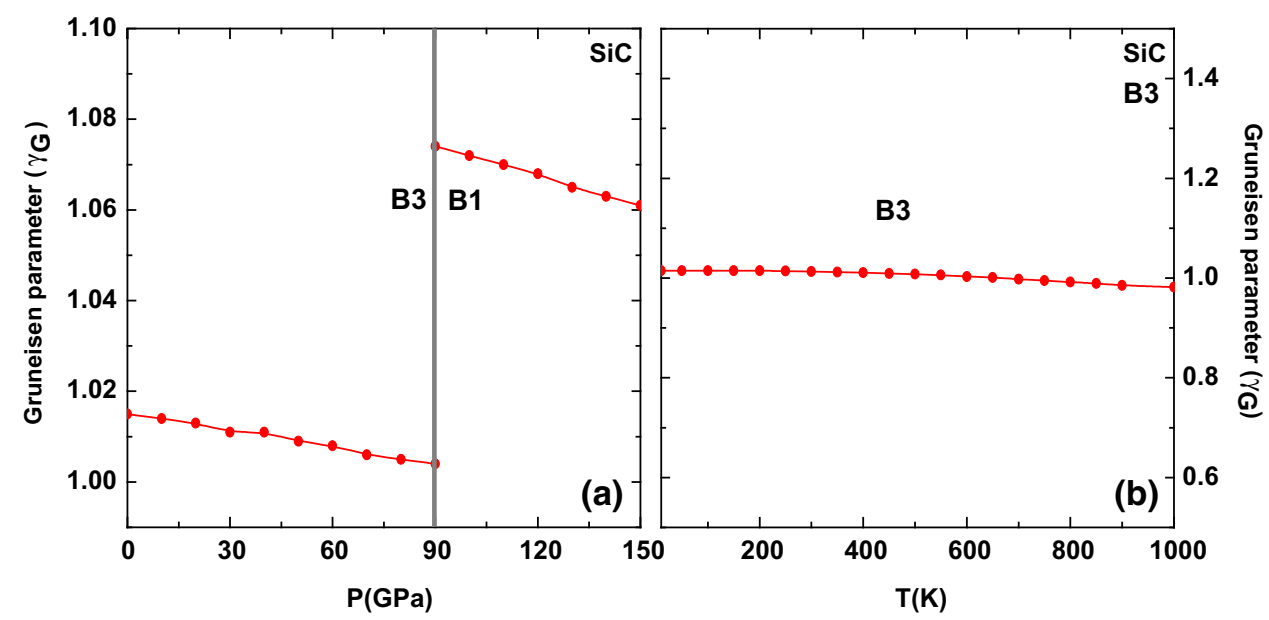

Fig. 13 Variation of isothermal compressibility $(\beta)$ with pressure and temperature

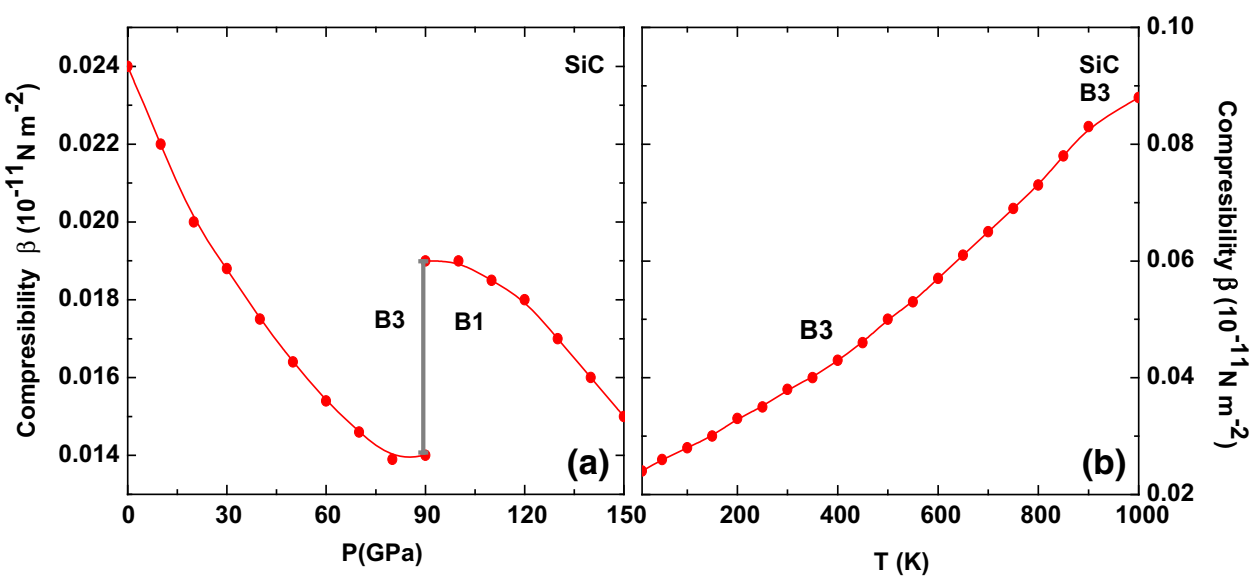


Fig. 14 Variation of Debye temperature $\left(\theta_{\mathrm{D}}\right)$ as a function of pressure and temperature

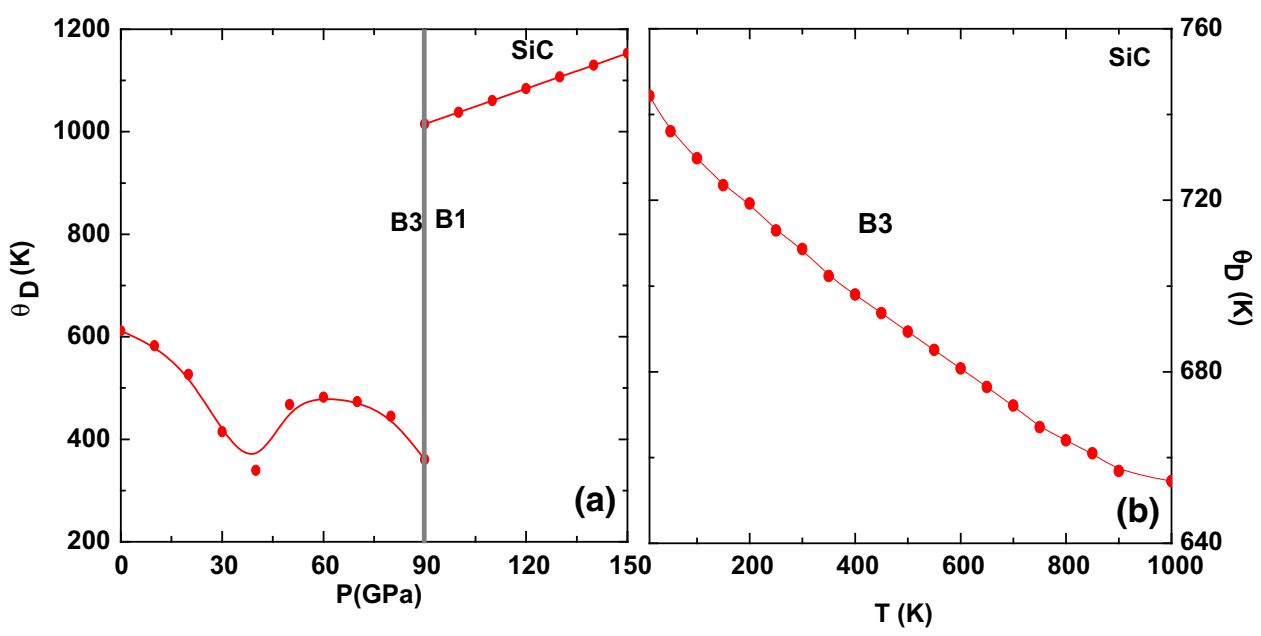

force constants induced by pressure decreases $\theta_{\mathrm{D}}$ in $B 3$ phase and after transition pressure it starts increasing which drives the system effectively towards the softening of lattice with increasing pressure.

Figure $14 \mathrm{~b}$ displays the variation of the Debye temperature as functions of temperature at zero pressure. Starting from zero temperature, $\theta_{\mathrm{D}}$ decreases rapidly. On the other hand, $\theta_{\mathrm{D}}$ enhances with applied pressures at zero temperature. It is worth commenting from high pressure and high temperature Debye temperature behaviour that (a) the pressure-dependent Debye temperature infers the mechanical stiffened bulk modulus due to $\mathrm{Si}-\mathrm{Si}, \mathrm{C}-\mathrm{C}$, and $\mathrm{Si}-\mathrm{C}$ bond compression and bond strengthening due to lattice vibration and (b) the thermal softening of bulk modulus results from bond expansion and bond weakening due to thermal stress in $3 \mathrm{C} \mathrm{SiC}$ ceramics in $\mathrm{ZB}$ structure.

A comparison of values of $\theta_{\mathrm{D}}(P)$ and $\theta_{\mathrm{D}}(T)$ at zero pressure and zero temperature results: $\theta_{\mathrm{D}}(T)>\theta_{\mathrm{D}}(P)$ implying the susceptibility of $\theta_{\mathrm{D}}$ with temperature. It is worth to comment that $\theta_{\mathrm{D}}(P)$ and $\theta_{\mathrm{D}}(T)$ behave differently with applied pressure and temperatures in $3 C \mathrm{SiC}$ ceramics. This is attributed to the fact vibrational spectrum of $\mathrm{SiC}$ lattice is controlled by aggregate elastic constants $C_{i j}$ behaviour. Thus, the understanding of Debye temperature behaviour of a material not only provides essential features of the vibrational spectrum but is also mandatory for technological and engineering applications. This quantity is useful as a reference for future experimental studies.

The cumulative effect of both pressure and temperature led us to define aggregate elastic constants $C_{a v}$ as

$C_{\mathrm{av} .}=\left(\frac{8 \pi}{3.15}\right)^{\frac{2}{3}}\left(\frac{k_{\mathrm{B}}}{h}\right)^{2}\left(\frac{M}{r}\right) \theta_{\mathrm{D}}^{2}$,

This is useful once Debye temperature at zero pressure is known from experiments. Despite various investigations on mechanical and thermodynamical properties, basic properties controlled by elastic constants related with high temperature behaviours are lacking.

In order to further explore the role of anharmonic effects in terms of heat capacity at constant volume $C_{v}$ and thermal expansion coefficient $\alpha$, we express the vibration term $A_{\text {vib. }}$ $[85,86]$ :

$A_{\text {vib. }}\left(\theta_{\mathrm{D}}, T\right)=n k_{\mathrm{B}} T\left[\frac{9 \theta_{\mathrm{D}}}{8 T}+3 \ln \left\{1-\exp \left(-\frac{\theta_{\mathrm{D}}}{T}\right)\right\}-D\left(\theta_{\mathrm{D}} / T\right)\right]$

The non-equilibrium Gibbs function, $G^{*}(V ; P, T)$, is minimized with respect to volume $V$ as

$\left[\frac{\partial G^{*}(V ; P, T)}{\partial V}\right]_{P, T}=0$

From Eq. (47), we determine heat capacity at constant volume $C_{v}$ as

$C_{v}=3 n k_{\mathrm{B}}\left[4 D\left(\frac{\theta_{\mathrm{D}}}{T}\right)-\frac{3 \theta_{\mathrm{D}} / T}{e^{\theta_{\mathrm{D}} / T}-1}\right]$

Figure 15 a documents the variations of heat capacity at constant volume, $C_{v}$, with pressure $P$ for both $B 3$ and $B 1$ phase of $\mathrm{SiC}$ at $T=600,800,1000$ and $1200 \mathrm{~K}$. The normalized heat capacity is $\left[C_{v}(P)-C_{v}(0)\right] / C_{v}(0)$, where $C_{v}(P)$ and $C_{v}(0)$ are heat capacity at any pressure $P$ and at zero pressure. Starting from zero pressure, normalized heat capacity initially increases and with further enhanced pressure it decreases abruptly in $B 1$ phase. This behaviour essentially points to the fact that the SiC lattice vibrations energy are controlled by both pressure as well temperature. Note that for higher temperatures $T \rightarrow \theta_{\mathrm{D}}$, the variation in heat capacity with pressure is weak in $B 1$ phase. Also, at $P_{T}$, reduced jump in between $\mathrm{ZB}$ and RS structures can be seen as compared to low temperatures.

The Debye temperature calculated from elastic constants must have a close resemblance to that measured from 
Fig. 15 Variation of heat capacity $\left(C_{v}\right)$ with pressure at different temperatures and with temperature at different pressures

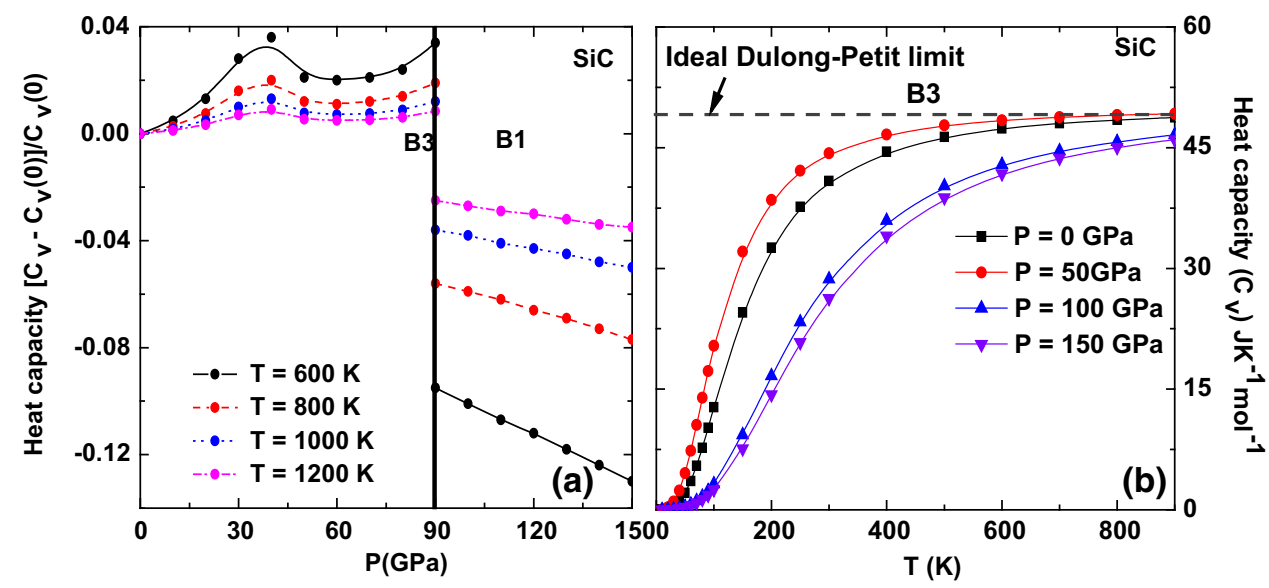

specific heat only at low temperatures. It is instructive to study the temperature-dependent behaviour of heat capacity. Figure $15 \mathrm{~b}$ illustrates the heat capacity at constant volume, $C_{v}$, behaviour as functions of temperature for various pressures $[0,50,100,150 \mathrm{GPa}]$ within the framework of quasi-harmonic model for $3 C \mathrm{SiC}$ ceramics. It can be seen that below room temperature $(300 \mathrm{~K}), C_{v}$ increases very rapidly with the temperature at all pressures. Above room temperature, $C_{v}$ increases slowly with the temperature. It almost approaches a constant ideal gas limit, the Dulong-Petit limit, $C_{v}(T)=3 R$, at higher temperatures as well at all pressures for $\mathrm{SiC}$ ceramics.

We further determine thermal expansion coefficient $\alpha_{\text {th.exp. using Eq. (47) as }}$

$\alpha_{\text {th.exp. }}=\frac{\gamma C_{v}}{B_{T} V}$

Apart from the pressure dependence of heat capacity at constant volume $C_{v}$, the Gruneisen parameter and Bulk modulus are also needed to elucidate the thermal expansion coefficient $\left(\alpha_{\text {th.exp. }}\right)$. It is a measure of any alteration in phonon frequency depending on the lattice's expansion or contraction in volume as a result of temperature variation. Figure 16a illustrates the pressure dependence of $\alpha_{\text {th.exp. }}$ for $3 C \mathrm{SiC}$ in both $\mathrm{ZB}$ and $\mathrm{RS}$ i.e., $B 3$ and $B 1$ phase. A nonlinear decrease in $\alpha_{\text {th.exp., }}$ is thus witnessed with pressure in both $\mathrm{ZnS}$ and $\mathrm{NaCl}$ phase. The decrease is more rapid in $\mathrm{ZB}$ structure while a slow decrease is documented in $\mathrm{RS}$ structure. We note that at $P_{T}$, the thermal expansion coefficient $\alpha_{\text {th.exp. }}$ is suppressed by $26-22 \%$ in SiC, at temperatures $600,800,1000$, and $1200 \mathrm{~K}$, respectively.

The variations of $\alpha_{\text {th.exp. }}$ as functions of temperature at various pressures have been plotted in Fig. $16 \mathrm{~b}$ for $3 \mathrm{C} \mathrm{SiC}$ ceramics. This figure shows that at low temperatures $(T<250 \mathrm{~K}), \alpha_{\text {th.exp. }}$ enhances rapidly with temperature at $P=0$ and $50 \mathrm{GPa}$. At high temperatures $(T>250 \mathrm{~K})$, a sharp increase of $\alpha_{\text {th.exp }}$ is witnessed at all pressures. This figure also suggests that with enhanced pressure, the increase of $\alpha_{\text {th.exp. }}$ with temperature becomes smaller. In other words, the slope of $\alpha_{\text {th.exp. }}$ gradually decreases at higher temperatures at all pressures except at $P=0 \mathrm{GPa}$. Returning back to pressure dependence of thermal expansion coefficient $\left(\alpha_{\text {th.exp. }}\right)$, we note a rapid decrease in ZB structure with the increase of pressure. A comparison of $\alpha_{\text {th.exp. }}(P)$ and $\alpha_{\text {th.exp. }}(T)$ reveals that $\alpha_{\text {th.exp. }}(P)$ values are smaller than that $\alpha_{\text {th.exp. }}(T)$ for SiC under same pressure and temperature. Thus, $\alpha_{\text {th.exp. }}(P)$ and $\alpha_{\text {th.exp. }}(T)$ are differently sensitive to the $\mathrm{Si}$ and $\mathrm{C}$ ions in $3 C \mathrm{SiC}$ ceramics as $\mathrm{SiC}$ lattice is mechanical hard due to bond strengthening and thermal soft due to bond weakening.

\section{Concluding remarks}

The present study addresses for the first time, the high pressure and high temperature-dependent structural, elastic, and thermodynamical studies in $\mathrm{ZB}$ and $\mathrm{RS}$ structure of $3 C \mathrm{SiC}$ ceramics. We have formulated an interatomic pairwise potential that incorporates the long-range Coulomb with charge transfer interactions, covalent nature of bonds, zero point energy effects and the short-range interactions as charge dipole-dipole and charge dipolequadruple (van der Waals), as well overlap repulsive interaction up to second-neighbour ions.

From the knowledge of Gibbs's free energies in ZB and RS structure, we determine the pressure-induced first-order structural phase transition of $\mathrm{SiC}$ at about $90 \mathrm{GPa}$. As a next step, we determine the cohesive energy as $6.301 \mathrm{eV}$ for $3 C \mathrm{SiC}$ ceramics and the volume collapse is about $13.8 \%$. Compressions in $\mathrm{SiC}$ at higher pressure indicate the mechanical stiffening of lattice. The phase transition pressure and volume collapse are consistent with earlier observations. We emphasize that agreement with experimental and theoretical data is not fortuitous, but it is 
Fig. 16 Variation of the thermal expansion coefficient $\left(\alpha_{\text {th.exp. }}\right)$ with pressure at different temperatures and with temperature at different pressures

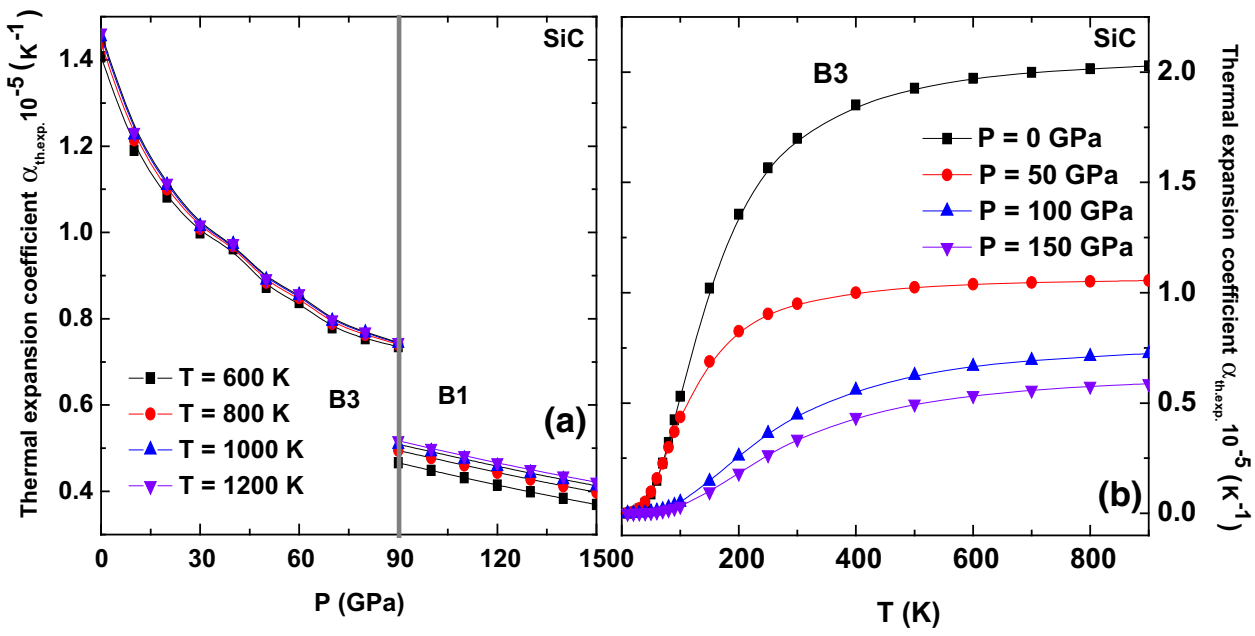

transfer and covalency) interaction and substantial anharmonic effects at high pressures.

(c) Elastic anisotropic parameter $\gamma_{1}^{2}$ in cubic $\mathrm{SiC}$ is negative and largely uninfluenced by application of pressure.

(d) Melting temperature $\left(T_{\mathrm{M}}\right)$, Vicker's hardness $\left(H_{V}\right)$, shear modulus $\left(G_{H}\right)$, Young's modulus $(E)$ and bulk modulus $\left(B_{T}\right)$ increase with enhanced pressure showing the hardening or stiffening of the lattice, and suppressed $T_{M}$, $G_{H}, E$ and $B_{T}$ variations in temperature suggest the weakening of the lattice as a result of thermal softening of SiC lattice.

(e) From the Pugh's ratio $(\phi)$ we classify $3 C \mathrm{SiC}$ as ductile material while to that Poisson's ratio suggest its brittle nature. The two empirical rules only differ on the exact border between the two types of behaviour and $\mathrm{SiC}$ is a borderline case between the classes of ductile and brittle materials.

(f) Grüneisen parameter $\left(\gamma_{\mathrm{G}}\right)$ Debye temperature $\left(\theta_{\mathrm{D}}\right)$, isothermal compressibility $(\beta)$, heat capacity at constant volume $\left(C_{v}\right)$ and thermal expansion coefficient $\left(\alpha_{\text {th.exp. }}\right)$ probe the importance of anharmonicity in $\mathrm{SiC}$ at heigh pressures and temperatures.

(g) Elastic properties showed $T_{\mathrm{M}}(P)<T_{\mathrm{M}}(T), H_{V}(P)$ $<H_{V}(T), E(P)<E(T), E(P)<E(T), \theta_{\mathrm{D}}(P)<\theta_{\mathrm{D}}(T)$ and $\alpha_{\text {th.exp. }}(P)<\alpha_{\text {th.exp. }}(T)$ infers that these are differently sensitive to the $\mathrm{Si}$ and $\mathrm{C}$ ions as $\mathrm{SiC}$ lattice is mechanical hard due to bond strengthening and thermal soft due to bond weakening.

To an end, an interatomic pairwise potential for cubic $\mathrm{SiC}$ incorporating the non-central many body forces as long-range Coulomb with charge transfer interactions, covalent nature of bonds (due to $\mathrm{Si}-\mathrm{Si}, \mathrm{Si}-\mathrm{C}$, and $\mathrm{C}-\mathrm{C}$ interacting electric fields), quantum effects, charge (b) Larger deviation in Cauchy discrepancy $\Delta_{1}^{2}$ emphasizes the importance of the many body non-central (charge 
dipole-dipole and charge dipole-quadruple (van der Waals), and the short-range interactions as overlap repulsion up to second-neighbour ions successfully explains the pressure and temperature induced structural, elastic and thermodynamical properties of $3 C \mathrm{SiC}$ ceramics consistent with available results.

Open Access This article is distributed under the terms of the Creative Commons Attribution 4.0 International License (http://creative commons.org/licenses/by/4.0/), which permits unrestricted use, distribution, and reproduction in any medium, provided you give appropriate credit to the original author(s) and the source, provide a link to the Creative Commons license, and indicate if changes were made.

\section{Appendix 1}

The relevant expressions for the aggregate second-order elastic constants (SOECs), and the pressure derivatives of SOECs are expressed for zinc-blende structure as

$$
\begin{aligned}
C_{11}= & L\left[0.2477 Z\left(Z+8 f\left(r_{0}\right)\right)+\frac{1}{3}\left(A_{1}+2 B_{1}\right)\right. \\
& \left.+\frac{1}{2}\left(A_{2}+B_{2}\right)+5.8243 Z a f^{\prime}\left(r_{0}\right)\right], \\
C_{12}= & L\left[-2.6458 Z\left(Z+8 f\left(r_{0}\right)\right)+\frac{1}{3}\left(A_{1}-4 B_{1}\right)\right. \\
& \left.+\frac{1}{4}\left(A_{2}-5 B_{2}\right)+5.8243 Z a f^{\prime}\left(r_{0}\right)\right], \\
C_{44}= & L\left[-0.123 Z\left(Z+8 f\left(r_{0}\right)\right)+\frac{1}{3}\left(A_{1}+2 B_{1}\right)+\frac{1}{4}\left(A_{2}+3 B_{2}\right)\right. \\
& \left.-\frac{1}{3} \nabla\left(-7.53912 Z\left(Z+8 f\left(r_{0}\right)\right)+A_{1}-B_{1}\right)\right]
\end{aligned}
$$

and

$B_{1}+B_{2}=-1.261 Z[Z+8 f(r)]$.

Thus, second-order elastic constants difference lead to finite value at Cauchy pressure:

$C_{12}-C_{44} \neq 0$

Henceforth, polarizability of the ions has effect on the elastic constants. It should be noted that if charge transfer mechanism is not taken into account, Cauchy relation: $C_{12}-C_{44}=0$, The Cauchy violations $\left(C_{12} \neq C_{44}\right)$ is seen by several crystals due to anisotropy in the electron distribution or angle bending. Here, $C_{11}$ represents a measure of resistance to deformation by applied stress and $C_{44}$ represents the measure of resistance to deformation with respect to applied shearing stress. The elastic constants $C_{12}$ and $C_{44}$ are related to the elasticity in shape, which is a shear constant.

The pressure derivatives of second-order elastic constants under hydrostatic pressure $P$ are obtained in the form

$$
\begin{aligned}
3 \Omega \frac{\mathrm{d} B_{T}}{\mathrm{~d} p}= & -\left[20.1788 Z\left(Z+8 f\left(r_{0}\right)\right)-3\left(A_{1}+A_{2}\right)\right. \\
& +4\left(B_{1}+B_{2}\right)+3\left(C_{1}+C_{2}\right)-104.8433 Z a f^{\prime}\left(r_{0}\right) \\
& \left.+22.7008 Z a^{2} f^{\prime \prime}\left(r_{0}\right)\right] \\
2 \Omega \frac{\mathrm{d} \sigma}{\mathrm{d} p}=- & {\left[-11.5756 Z\left(Z+8 f\left(r_{0}\right)\right)+2\left(A_{1}-2 B_{1}\right)\right.} \\
& \left.+\frac{3}{2} A_{2}-\frac{7}{2} B_{2}+\frac{1}{4} C_{2}+37.5220 Z a f^{\prime}\left(r_{0}\right)\right]
\end{aligned}
$$

and

$$
\begin{aligned}
\Omega \frac{\mathrm{d} C_{44}}{\mathrm{~d} p}= & -\left[\begin{array}{l}
0.4952 Z\left(Z+8 f\left(r_{0}\right)\right)+\frac{1}{3}\left(A_{1}-4 B_{1}+C_{1}\right) r \\
+\frac{1}{4}\left(2 A_{2}-6 B_{2}+C_{2}\right)+4.9667 Z a f^{\prime}\left(r_{0}\right)+2.522 Z a^{2} f^{\prime \prime}\left(r_{0}\right)
\end{array}\right] \\
& +\nabla\left[-17.5913 Z\left(Z+8 f\left(r_{0}\right)\right)+A_{1}-B_{2}-\frac{2}{3} C_{11}\right. \\
& \left.+40.6461 Z a f^{\prime}\left(r_{0}\right)-5.044 Z a^{2} f^{\prime \prime}\left(r_{0}\right)\right] \\
& +\nabla^{2}\left[3 . 1 4 1 6 Z \left(Z+8 f\left(r_{0}\right)+\frac{2}{3}\left(A_{1}-B_{1}\right)\right.\right. \\
& \left.+\frac{C_{1}}{3}-15.9412 Z a f^{\prime}\left(r_{0}\right)+8.8052 Z a^{2} f^{\prime \prime}\left(r_{0}\right)\right]
\end{aligned}
$$

The notations are

$$
\begin{aligned}
& \Omega=-5.0440 g+A_{1}+A_{2}-2\left(B_{1}+B_{2}\right)+17.4730 Z g_{1} \\
& \nabla=\left[\frac{-7.5391 Z\left(Z+8 f\left(r_{0}\right)\right)+\left(A_{1}-B_{1}\right)}{-3.141 Z\left(Z+8 f\left(r_{0}\right)\right)+\left(A_{1}+2 B_{1}\right)+21.765 Z^{\prime} f^{\prime}\left(r_{0}\right)}\right],
\end{aligned}
$$

Finally, the anharmonic third-order elastic constants (TOECs) for zinc-blende structure are as follows:

$$
\begin{aligned}
C_{111}= & \frac{e^{2}}{4 a^{4}}\left[0.5184 g+\frac{1}{9}\left(C_{1}-6 B_{1}-3 A_{1}\right)+\frac{1}{4}\left(C_{2}-B_{2}-3 A_{2}\right)\right. \\
& \left.-2\left(B_{1}+B_{1}\right)-9.9326 Z g_{1}+2.522 Z g_{2}\right], \\
C_{112}= & \frac{e^{2}}{4 a^{4}}\left[0.3828 g+\frac{1}{9}\left(C_{1}+3 B_{1}-3 A_{1}\right)+\frac{1}{8}\left(C_{2}+3 B_{2}-3 A_{2}\right)\right], \\
& -11.642 Z g_{1}+2.522 Z g_{2} \\
C_{123}= & \frac{e^{2}}{4 a^{4}}\left[6.1585 g+\frac{1}{9}\left(C_{1}+3 B_{1}-3 A_{1}\right)-12.5060 Z g_{1}+2.5220 Z g_{2}\right], \\
C_{144}= & \frac{e^{2}}{4 a^{4}}\left\{6.1585 g+\frac{1}{9}\left(C_{1}+3 B_{1}-3 A_{1}\right)-4.1681 Z_{1}+.8407 z g_{2}+\right. \\
+ & \nabla\left[-3.3507 g-\frac{2}{9} C_{1}+13.5486 Z g_{1}-1.681 g_{2}\right] \\
+ & \left.\nabla^{2}\left[-1.5637 g+\frac{2}{3}\left(A_{1}-B_{1}\right)+\frac{C_{1}}{9}-5.3138 Z g_{1}+2.9350 Z g_{2}\right]\right\}
\end{aligned}
$$




$$
\begin{aligned}
C_{166}= & \frac{e^{2}}{4 a^{4}}\left\{-2.1392 g+\frac{1}{9}\left(C_{1}-6 B_{1}-3 A_{1}\right)+\frac{1}{8}\left(C_{2}-5 B_{2}-3 A_{2}\right)-\left(B_{1}-B_{2}\right)\right. \\
& -4.168 Z g_{1}+.8407 Z g_{2}+\nabla\left[-8.3768 g+\frac{2}{3}\left(A_{1}-A_{2}\right)-\frac{2}{9} C_{1}+13.5486 Z g_{1}-1.6813 Z g_{2}\right] \\
& +\nabla^{2}\left[2.3527 g+\frac{C_{1}}{9}-5.3138 Z g_{1}+2.9350 Z g_{2}\right] \\
C_{456}= & \frac{e^{2}}{4 a^{4}}\left\{4.897 g+\frac{1}{9}\left(C_{1}-6 B_{1}-3 A_{1}\right)-B_{2}+\nabla\left[-5.0261 g-\frac{1}{9} C_{1}\right]\right. \\
& \left.+\nabla^{2}\left[7.0580 g+\frac{1}{3} C_{1}\right]+\nabla^{3}\left[-4.8008 g+\frac{1}{3}\left(A_{1}-B_{1}\right)-\frac{1}{9} C_{1}\right]\right\}
\end{aligned}
$$

In view of equilibrium condition, the third-order elastic constants difference also lead to finite value at Cauchy pressure inferring that polarizability of the ions has effect on the elastic constants:

$$
\begin{aligned}
& C_{112}-C_{166} \neq 0 \\
& C_{123}-C_{456} \neq 0 \\
& C_{144}-C_{456} \neq 0 \\
& C_{123}-C_{144} \neq 0
\end{aligned}
$$

Furthermore, the third-order elastic constants satisfy the identity:

$C_{123}+2 C_{456}-3 C_{144}=0$

Various symbols appear in the earlier expressions are associated with the crystal energy and have been defined below:

$$
\begin{aligned}
& A_{1}=A_{i j}=\frac{4 a^{3}}{e^{2}}\left(\frac{\mathrm{d}^{2}}{\mathrm{~d} r^{2}} V_{i j}(r)\right)_{r=a}, \\
& A_{2}=A_{i i}=A_{j j}=\frac{4 a^{3}}{e^{2}}\left(\frac{\mathrm{d}^{2}}{\mathrm{~d} r^{2}} V_{i i}(r)+\frac{\mathrm{d}^{2}}{\mathrm{~d} r^{2}} V_{j j}(r)\right)_{r=\sqrt{3} a / 4} \\
& B_{1}=B_{i j}=\frac{4 a^{2}}{e^{2}}\left(\frac{\mathrm{d}}{\mathrm{d} r} V_{i j}(r)\right)_{r=a}, \\
& B_{2}=B_{i i}=B_{j j}=\frac{4 a^{2}}{e^{2}}\left(\frac{\mathrm{d}}{\mathrm{d} r} V_{i i}(r)+\frac{\mathrm{d}}{\mathrm{d} r} V_{j j}(r)\right)_{r=\sqrt{3} a / 4} \\
& C_{1}=C_{i j}=\frac{4 a^{4}}{e^{2}}\left(\frac{\mathrm{d}^{3}}{\mathrm{~d} r^{3}} V_{i j}(r)\right)_{r=a}, \\
& C_{2}=C_{i i}=C_{j j}=\frac{4 a^{4}}{e^{2}}\left(\frac{\mathrm{d}^{3}}{\mathrm{~d} r^{3}} V_{i i}(r)+\frac{\mathrm{d}^{3}}{\mathrm{~d} r^{3}} V_{j j}(r)\right)_{r=a}, \\
& g=Z+8 f(r)
\end{aligned}
$$

$$
g_{1}=r_{o} \mathrm{~d} f(r)
$$

$g(2)=r_{o} d \mathrm{~d} f(r)$

Similarly, the expressions for the second-order elastic constants for RS structure crystals are as follows:

$C_{11}=\frac{e^{2}}{4 a^{4}}\left\{-5.112 Z_{m}^{2}+A_{1}+\frac{A_{2}+B_{2}}{2}+9.3204 Z\left(a f^{\prime}(r)\right)\right\}$

$C_{12}=\frac{e^{2}}{4 a^{4}}\left\{0.226 Z_{m}^{2}-B_{1}+\frac{A_{2}-5 B_{2}}{4}+9.3204 Z\left(a f^{\prime}(r)\right)\right\}$

$C_{44}=\frac{e^{2}}{4 a^{4}}\left\{2.556 Z_{m}^{2}+B_{1}+\frac{A_{2}+3 B_{2}}{4}\right\}$

and

$B_{1}+B_{2}=-1.165 Z[Z+12 f(r)]$.

For non-central many body forces as we deal with the second-order elastic constants difference lead to finite value at Cauchy pressure:

$C_{12}-C_{44} \neq 0$

The pressure derivatives of second-order aggregate elastic constants under hydrostatic pressure $P$ are obtained in the form

$$
\begin{aligned}
3 \Omega \frac{\mathrm{d} B_{T}}{\mathrm{~d} p}= & -\left\{13.980 Z_{m}^{2}+C_{1}-3 A_{1}+C_{2}-3 A_{2}\right. \\
& \left.-167.7648 Z\left(a f^{\prime}(r)\right)+41.9420 Z\left(a^{2} f^{\prime \prime}(r)\right)\right\} \\
2 \Omega \frac{\mathrm{d} \sigma}{\mathrm{d} p}= & -\left\{23.682 Z_{m}^{2}+C_{1}+\frac{C_{2}+6 A_{2}-6 B_{2}}{4}\right. \\
& \left.-50.0752 Z\left(a f^{\prime}(r)\right)+13.9808 Z\left(a^{2} f^{\prime \prime}(r)\right)\right\}
\end{aligned}
$$




$$
\begin{aligned}
& \Omega \frac{\mathrm{d} C_{44}}{\mathrm{~d} p}=-\left\{-11.389 Z_{m}^{2}+A_{1}-3 B_{1}\right. \\
& \left.\quad+\frac{C_{2}+2 A_{2}-10 B_{2}}{4}+44.6528 Z\left(a f^{\prime}(r)\right)\right\}
\end{aligned}
$$

The notation $\Omega$ is

$$
\Omega=-2.330 Z_{m}^{2}+A_{1}+A_{2}+27.9612 Z\left(a f^{\prime}(r)\right)
$$

Finally, the anharmonic third-order elastic constants for RS structure are as follows:

$$
\begin{aligned}
C_{111}= & \frac{e^{2}}{4 a^{4}}\left\{37.556 Z_{m}^{2}+C_{1}-3 A_{1}-\frac{3 A_{2}+9 B_{2}-C_{2}}{4}\right. \\
& \left.-89.305 Z\left(a f^{\prime}(r)\right)+13.980 Z\left(a^{2} f^{\prime \prime}(r)\right)\right\} \\
C_{112}= & \frac{e^{2}}{4 a^{4}}\left\{-4.836 Z_{m}^{2}-\frac{3 A_{2}+3 B_{2}-C_{2}}{8}\right. \\
& \left.-18.640 Z\left(a f^{\prime}(r)\right)+4.66 Z\left(a^{2} f^{\prime \prime}(r)\right)\right\} \\
C_{123}= & \frac{e^{2}}{4 a^{4}}\left\{2.717 Z_{m}^{2}+16.692 Z\left(a f^{\prime}(r)\right)\right\} \\
C_{144}= & \frac{e^{2}}{4 a^{4}}\left\{2.717 Z_{m}^{2}+5.564 Z\left(a f^{\prime}(r)\right)\right\} \\
C_{166}= & \frac{e^{2}}{4 a^{4}}\left\{-4.836 Z_{m}^{2}-2\left(B_{1}+B_{2}\right)\right. \\
& \left.-\frac{3 A_{2}-3 B_{2}-C_{2}}{8}+5.564 Z\left(a f^{\prime}(r)\right)\right\} \\
C_{456}= & \frac{e^{2}}{4 a^{4}}\left\{2.717 Z_{m}^{2}-\left(B_{1}+B_{2}\right)\right\}
\end{aligned}
$$

Various symbols appear in the above expressions are associated with the crystal energy and have the following form in RS structure

$$
\begin{aligned}
& A_{1}=\frac{8 a^{3}}{e^{2}}\left[\frac{\mathrm{d}^{2}}{\mathrm{~d} r^{2}} V_{i j}(r)\right]_{r=a}, \\
& A_{2}=\frac{16 a^{3}}{e^{2}}\left[\frac{\mathrm{d}^{2}}{\mathrm{~d} r^{2}} V_{i i}(r)+\frac{\mathrm{d}^{2}}{\mathrm{~d} r^{2}} V_{j j}(r)\right]_{r=\sqrt{2} a}, \\
& B_{1}=\frac{8 a^{3}}{e^{2}}\left[\frac{1}{r} \frac{\mathrm{d}}{\mathrm{d} r} V_{i j}(r)\right]_{r=a}, \\
& B_{2}=\frac{16 a^{3}}{e^{2}}\left[\frac{1}{r} \frac{\mathrm{d}}{\mathrm{d} r} V_{i i}(r)+\frac{1}{r} \frac{\mathrm{d}}{\mathrm{d} r} V_{j j}(r)\right]_{r=\sqrt{2} a}, \\
& C_{1}=\frac{8 a^{3}}{e^{2}}\left[r \frac{\mathrm{d}^{3}}{\mathrm{~d} r^{3}} V_{i j}(r)\right]_{r=a}, \\
& C_{2}=\frac{16 a^{3}}{e^{2}}\left[r \frac{\mathrm{d}^{3}}{\mathrm{~d} r^{3}} V_{i i}(r)+\frac{\mathrm{d}^{3}}{\mathrm{~d} r^{3}} V_{j j}(r)\right]_{r=\sqrt{2} a}
\end{aligned}
$$

in terms of the short-range energy

$$
V_{i j}(r)=\sum_{i j} b \beta_{i j} \exp \left(\frac{r_{i}+r_{j}-r_{i j}}{\rho}\right)-\sum_{i j} \frac{C}{r_{i j}^{6}}-\sum_{i j} \frac{D}{r_{i j}^{8}}
$$

The short-range interaction (SR) energy is expressed in terms of the overlap repulsion (first term) and the induced charge dipole-dipole and charge dipolequadruple (van der Waals) [second and third terms], respectively.

\section{Appendix 2}

The temperature-dependent elastic, thermal and thermodynamical properties of $\mathrm{SiC}$ are now studied within the quasi-harmonic Debye approximation. The Helmholtz free energy at temperature below the melting point of $\mathrm{SiC}$ ceramics in the quasi-harmonic model is applicable to evaluate equation of state and thus the elastic properties. The non-equilibrium Gibbs's free energy function, $G^{*}(V$; $P, \quad T)$ within the quasi-harmonic approximation is $G=U+P V+A_{\text {vib. }}\left[\theta_{\mathrm{D}}(V) ; T\right]$.

In order to determine the anharmonic effects on the elastic constants at higher temperatures, we use the equation of state (EOS) as:

$P=-\frac{\mathrm{d} U}{\mathrm{~d} V}+T \alpha_{v} B_{T}$

Here, $P$ is the pressure, $T$ the temperature, $U$ the total potential energy, $\alpha_{v}$ the volume thermal expansion coefficient, $B_{T}$ the isothermal bulk modulus. The second term in Eq. (106) is the thermal phonon pressure. The temperaturedependent second-order elastic constants $C_{i j}$ are derived from the dynamical matrix of modified Rigid Shell model [19-26, 35-42] and the method of long waves as

$$
\begin{aligned}
C_{11}= & 1.573 T \alpha_{v} B_{T} \\
& +\frac{e^{2}}{4 a^{4}}\left\{\frac{1}{3} A_{1}-0.9 B_{1}-1.573 B_{2}+8.9558\left(a f^{\prime}(r)\right)\right\}
\end{aligned}
$$

$$
\begin{aligned}
C_{12}= & -1.786 T \alpha_{\nu} B_{T} \\
& +\frac{e^{2}}{4 a^{4}}\left\{\frac{1}{3} A_{1}+1.45 B_{1}+1.786 B_{2}+8.9558\left(a f^{\prime}(r)\right)\right\} \\
C_{44}= & -0.786 T \alpha_{v} B_{T} \\
& +\frac{e^{2}}{4 a^{4}}\left\{\frac{1}{3} A_{1}+1.45 B_{1}+1.786 B_{2}-\frac{1}{3} \Delta\right\}
\end{aligned}
$$

with 


$$
\begin{aligned}
\Delta= & {\left[A_{1}+6.77 B_{1}+7.78 B_{2}-7.77\left\{T \alpha_{\nu} B_{T} \frac{4 a^{4}}{e^{2}}\right\}\left\{\left(A_{1}+2 B_{1}\right)\right.\right.} \\
& \left.\left.+3\left(A_{2}+2 B_{2}\right)\right\}^{-1}\right]
\end{aligned}
$$

and

$$
T \alpha_{\nu} B_{T}=\frac{e^{2}}{4 a^{4}}\left[1.9395 Z\left[Z+8 f\left(r_{0}\right)\right]+\left(B_{1}+B_{2}\right)\right]
$$

Herein, the potential energy incorporates the long-range Coulomb with charge transfer interactions, covalent nature of bonds, and short-range overlap repulsive interaction up to second-neighbour as well charge dipole-dipole and charge dipole-quadruple (van der Waals) interaction.

We use equilibrium condition to obtain temperature dependence of hardness and range parameters as

$r\left|\frac{\mathrm{d} U(r)}{\mathrm{d} r}\right|_{r=r_{0}}=3 T \alpha_{v} V B_{T}$

Here, $V$ is the unit cell volume of $\mathrm{SiC}$.

\section{References}

1. Levinshteæin, M.E., Rumyantsev, S.L., Shur, M.: Properties of advanced semiconductor materials: GaN, AlN, InN, BN, SiC, SiGe. Wiley, New York (2001)

2. Parfenova, I.I.: Substitutional $3 \mathrm{~d}$ impurities in cubic silicon carbide. Semiconductors 38, 189 (2004)

3. Yoshida, M., Onodera, A., Ueno Takemura, M.K., Shimomura, O.: Pressure-induced phase transition in SiC. Phys. Rev. B 48, 10587 (1993)

4. Sekine, T., Kobayashi, T.: Shock compression of $6 H$ polytype $\mathrm{SiC}$ to $160 \mathrm{GPa}$. Phys. Rev. B 55, 803 (1997)

5. Chang, K.J., Cohen, M.L.: Ab initio pseudopotential study of structural and high-pressure properties of SiC. Phys. Rev. B 35, 8196 (1987)

6. Miao, M.S., Lambrecht, W.R.L.: Unified path for high-pressure transitions of $\mathrm{SiC}$ polytypes to the rocksalt structure. Phys. Rev. B 68, 92103 (2003)

7. Durandurdu, M., Drabold, D.A.: Ab initio simulations of the structural phase transformation of $2 \mathrm{H}-\mathrm{SiC}$ at high pressure. Phys. Rev. B 75, 235204 (2007)

8. Shimojo, F., Ebbsj, O.I., Lalia, R., Nakano, A., Rino, J.P., Vashista, P.: Molecular dynamics simulation of structural transformation in silicon carbide under pressure. Phys. Rev. Lett. 84, 3338 (2000)

9. Yu-Ping, L., Duan-Wei, H., Jun, Z., Xiang Dong, Y.: Firstprinciples study of pressure-induced phase transition in silicon carbide. Phys. B 403, 3543 (2008)

10. Vashishta, P., Kalia, R.K., Nakano, A.: Interaction potential for silicon carbide: A molecular dynamics study of elastic constants and vibrational density of states for crystalline and amorphous silicon carbide. J. Appl. Phys. 101, 103515 (2007)

11. Cheong, B.H.K., Chang, J., Cohen, M.L.: Pressure dependences of band gaps and optical-phonon frequency in cubic SiC. Phys. Rev. B 44, 1053 (1991)

12. Miao, M.S., Prikhodko, M., Lambrecht, W.R.L.: Changes of the geometry and band structure of $\mathrm{SiC}$ along the orthorhombic high- pressure transition path between the zinc-blende and rocksalt structures. Phys. Rev. B 66, 64107 (2002)

13. Miao, M.S., Prikhodko, M., Lambrecht, W.R.L.: Orthorhombic intermediate state in the zinc blende to rocksalt transformation path of SiC at high pressure. Phys. Rev. Lett. 88, 18960 (2002)

14. Durandurdu, M.: Pressure-induced phase transition of SiC. J. Phys. Condens. Matter 16, 4411-4417 (2004)

15. Stillinger, F.H., Weber, T.A.: Computer-simulation of local order in condensed phases of silicon. Phys. Rev. B 31, 5262 (1985)

16. Karch, K., Bechstedt, F., Pavone, P., Strauch, D.: Pressure-dependent properties of SiC polytypes. Phys. Rev. B 53, 13400 (1996)

17. Oganov, A.R. (ed.): Modern methods of crystal structure prediction (2011) Wiley VCH Verlag GmbH and Co. KGaA, Weinheim

18. Dion, M., Rydberg, H., Schroder, E., Langreth, D.C., Lundqvist, B.I.: Van der Waals density functional for general geometries. Phys. Rev. Lett. 92, 246401 (2004)

19. Varshney, D., Kaurav, N., Sharma, P., Shah, S., Singh, R.K.: Structural phase transition and elastic properties of $\mathrm{ZnSe}$ at high pressure. Phase Transit 77, 1075 (2004)

20. Varshney, D., Sharma, P., Kaurav, N., Shah, S., Singh, R.K.: Study of elastic properties and their pressure dependence of semi magnetic semiconductors. J. Phys. Soc. 74, 382 (2005)

21. Varshney, D., Kaurav, N., Kinge, R., Singh, R.K.: B1-B2 structural phase transition and elastic properties of $\mathrm{UX}(\mathrm{X}=\mathrm{S}$, Se and Te) compounds at high pressure. J. Phys. Condens. Matter 19, 236204 (2007)

22. Varshney, D., Sharma, U., Kaurav, N.: Structural phase transformation and mechanical properties of semimagnetic semiconductors $\mathrm{Zn}_{1-x} \mathrm{M}_{x} \mathrm{Se}(\mathrm{M}=\mathrm{Mn}, \mathrm{Fe}$ and $\mathrm{Cd})$. J. Phys. Condens. Matter 20, 075204 (2008)

23. Varshney, D., Joshi, G., Kaurav, N., Singh, R.K.: Structural phase transition (zincblende-rocksalt) and elastic properties in AlY $(\mathrm{Y}=\mathrm{N}, \mathrm{P}$ and As) compounds: pressure-induced effects. J. Phys. Chem. Solids 70, 451 (2009)

24. Varshney, D., Joshi, G.: High-pressure structural phase transition and elastic properties of $\mathrm{Ga}_{1-x} \operatorname{In}_{x} \mathrm{As}$ semiconducting compounds. Eur. Phys. J. B 70, 523 (2009)

25. Varshney, D., Joshi, G., Varshney, M., Shriya, S.: Pressure induced structural phase transition and elastic properties in BSb, AlSb, GaSb and InSb compounds. Phys. B 405, 1663 (2010)

26. Varshney, D., Joshi, G., Varshney, M., Shriya, S.: Pressure dependent elastic and structural (B 3-B1) properties of Ga based monopnictides. J. Alloys Compd. 495, 23 (2010)

27. Hafemeister, D.W., Flygare, W.H.: Outer-shell overlap integral as a function of distance for halogen-halogen, halogen-alkali, and alkali-alkali ions in the alkali halide lattices. J. Chem. Phys. Soc 43, 795 (1965)

28. Tosi, M.P.: Cohesion of ionic solids in the born model. Solid State Phys. 16, 1 (1964)

29. Born, M., Huang, K.: Dynamical Theory of Crystal Lattices. Clarendon, Oxford (1956)

30. Motida, K.: Szigeti charge and its correlation with hyperine coupling constant of doped $\mathrm{Mn}^{2+}$ ion in divalent metal compounds. J. Phys. Soc. Jpn. 49, 213 (1980)

31. Motida, K.: Effect of covalency on phonon dispersion relations in $\mathrm{NaCl}$ type alkali halide crystal. J. Phys. Soc. Jpn. 55, 1636 (1986)

32. Slater, J.C., Kirkwood, J.G.: The vander Waals forces in gases. Phys. Rev. 37, 682 (1931)

33. Lowdin, P.O.: Quantum theory of cohesive properties of solids. Adv. Phys. 5, 1 (1956)

34. Lundqvist, S.O.: Three body potential for alkali halides. Ark. Fys. 12, 263 (1957)

35. Varshney, D., Joshi, G., Varshney, M., Shriya, S.: Pressure induced mechanical properties of boron-based pnictides. Solid State Sci. 12, 864 (2010) 
36. Varshney, D., Dodiya, N., Shaikh, M.W.: Structural properties and electrical resistivity of Na-substituted lanthanum manganites: $\mathrm{La}_{1-x} \mathrm{Na}_{x} \mathrm{MnO}_{3+y}(x=0.1,0.125$ and 0.15). J. Alloys Compd. 509, 7447 (2011)

37. Varshney, D., Choudhary, D., Shaikh, M.W., Khan, E.: Electrical resistivity behaviour of sodium substituted manganites: electronphonon, electron-electron and electron-magnon interactions. Eur. Phys. J. B 76, 327 (2010)

38. Varshney, D., Kaurav, N., Kinge, R., Singh, R.K.: High-pressure induced structural phase transition in alkaline earth $\mathrm{CaX}(\mathrm{X}=\mathrm{S}$, Se and Te) semiconductors: NaCl-type (B1) to CsCl-type (B2). J. Alloys Compd. 484, 239 (2009)

39. Varshney, D., Kaurav, N., Kinge, R., Singh, R.K.: Pressure induced phase transition (B1-B2) and elastic properties in alkaline earth $\mathrm{BaX}(\mathrm{X}=\mathrm{S}, \mathrm{Se}$ and $\mathrm{Te})$ chalcogenides. Phase Transit. 81, 1 (2008)

40. Varshney, D., Kaurav, N., Kinge, R., Singh, R.K.: High pressure structural (B1-B2) phase transition and elastic properties of II-VI semiconducting Sr chalcogens. Comput. Mater. Sci. 41, 529 (2008)

41. Varshney, D., Kaurav, N., Sharma, U., Singh, R.K.: Pressure induced phase transition and elastic properties of $\mathrm{Y}$ and $\mathrm{Sc}$ antimonides. J. Alloys Compd. 448, 250 (2008)

42. Varshney, D., Kaurav, N., Kinge, R., Shah, S., Singh, R.K.: Study of elastic properties and their pressure dependence of lanthanum monochalcogenides. High Press. Res. 25, 145 (2005)

43. Tessman, J.R., Kahn, A.H., Shockley, W.: Electronic polarizabilities of ion in crystals. Phys. Rev. 92, 890 (1953)

44. Shannon, R.D.: Dielectric polarizabilities of ions in oxides and fluorides. J. Appl. Phys. 73, 348 (1993)

45. Weast, R.C. (ed.): Handbook of Chemistry and Physics, 63rd edn. CRC, BocaRaton (1982)

46. Aleksandrov, I.V., Goncharov, A.F., Stishov, S.M., Yakovenko.: Equation of state and Raman scattering in cubic $\mathrm{BN}$ and $\mathrm{SiC}$ at high pressure. EV, Pis'ma h.Eksp. Teor. Fiz. 50, 116 (1989) (JETP Lett. 50, 127 (1989))

47. Feldman, D.W., Parker, H., Choyke, W., Patric, L.: Phonon dispersion curves by Raman scattering in $\mathrm{SiC}$ polytypes $3 \mathrm{C}, 4 \mathrm{H}, 6 \mathrm{H}$, 15R, and 21 R. Phys. Rev. 173, 787 (1968)

48. Jun, L., Vohra, Y.K.: Raman modes of $6 \mathrm{H}$ polytype of silicon carbide to ultrahigh pressures: a comparison with silicon and diamond. Phys. Rev. Lett 72, 4105 (1994)

49. Wagman, D.D., Evans, W.H., Parker, V.B., Halow, E., Baily, S.M., Shumm, R.H.: Selected values of chemical thermodynamics properties, tables for the first thirty four elements in the standard order of arrangement, Natl. Bur. Std. (U.S.) Tech. Note No. 270-3 (U.S. GPO, Washington, DC, 1968)

50. Lambrecht, W.R.L., Segall, B., Methfessel, M., van Schilfgaarde, M.: Calculated elastic constants and deformation potentials of cubic SiC. Phys. Rev. B 44, 3685 (1991)

51. Murnaghan, F.D.: The compressibility of media under extreme, pressures. Proc. Natl. Acad. Sci. U. S. A. 3, 244 (1944)

52. Goel, P., Choudhury, N., Chaplot, S.L.: Superionic behavior of lithium oxide $\mathrm{Li}_{2} \mathrm{O}$ : a lattice dynamics and molecular dynamics study. Phys. Rev. B 70, 174307 (2004)

53. Xiao-Feng, Li, Xiang-Rong, Chen, Chuan-Min, Meng, Guang-Fu, $\mathrm{Ji}$ : Ab initio calculations of elastic constants and thermodynamic properties of $\mathrm{Li}_{2} \mathrm{O}$ for high temperatures and pressures. Solid State Commun. 139, 197-200 (2006)

54. Yun-Dong, G., Ze-Jin, Y., Qing-He, G., Zi-Jiang, L., Wei, D.: The phase transition, and elastic and thermodynamic properties of $\mathrm{CaS}$ derived from first-principles calculations. J. Phys. Condens. Matter 20, 115203 (2008)

55. Kunc, K., Balkanski, M.: Nusimovici MA Lattice dynamics of several A $N B 8-N$ compounds having the zincblende structure II. Numerical calculations. Phys. Status Solidi (b) 72, 229 (1975)
56. Lee, D.H., Joannopoulos, J.D.: Simple scheme for deriving atomic force constants. Application to SiC. Phys. Rev. Lett. 48, 1846 (1982)

57. Denteneer, P.J.H., van Haeringen, W.: Ground-state properties of polytypes of silicon carbide. Phys. Rev. B 33, 2831 (1986)

58. Tolpygo, K. B. Optical, elastic and piezoelectric properties of ionic and covalent with the $\mathrm{ZnS}$ type lattice. F. Tverd. Tela (Leningrad) 1960; 2:2655 Sov. Phys. Solid State 1961; 2:2367

59. Yean Jr, D.H., Riter, J.R.: Estimates of isothermal bulk moduli for group iva crystals with the zinc blende structure. J. Phys. Chem. Solids 32, 653 (1971)

60. Carnahan, R.D.J.: Elastic properties of silicon carbide. Am. Ceram. Soc. 51, 223 (1968)

61. Churcher, N., Kunc, K., Heine, V.: Calculated ground-state properties of silicon carbide. J. Phys. C 19, 4413 (1986)

62. Weber, W.J., Yu, N., Wang, L.M., Hess, N.J.: Temperature and dose dependence of ion-beam-induced amorphization in $\alpha$-SiC. J. Nucl. Mater. 244, 258 (1997)

63. Tang, M., Yip, S.: Lattice instability in $\beta-\mathrm{SiC}$ and simulation of brittle fracture. J. Appl. Phys. 76, 2719 (1994)

64. Weber, W.J., Yu, N., Wang, L.M., Hess, N.: Structure and properties of ion-beam-modified $(6 \mathrm{H})$ silicon carbide. J. Mater. Sci. Eng. A 253, 62 (1998)

65. Gao, F., Weber, W.J.: Mechanical properties and elastic constants due to damage accumulation and amorphization in SiC. Phys. Rev. B 69, 224108 (2004)

66. Vukcevich, M.R.: The elastic constants of cubic crystals with covalent and partially covalent bonds. Phys. Stat. Solidi (b) 54, 435 (1972)

67. Barsch, G.R.: Relation between third-order elastic constants of single crystals and polycrystals. J. Appl. Phys. 39, 3780 (1968)

68. Hill, R.: The elastic behavior of a crystalline aggregate. Proc. Phys. Soc. (Lond.) 65A, 349 (1952)

69. Voigt, W.: Lehrbuch der Kristallphysik. Teubner, Leipzig (1928)

70. Reuss, A.: Calculation of the flow limits of mixed crystals on the basis of the plasticity of monocrystals. Angew Z. Math. Mech. 9, 49 (1929)

71. Takahiro, M., Tetsuro, N., Yasuaki, N., Katsuhiro, K., Tastuya, Y., Hidetoshi, N., Satoshi, N.: Measurement of high-temperature elastic properties of ceramics using a laser ultrasonic method. J. Am. Ceram. Soc. 84, 1521-1525 (2001)

72. Pugh, S.F.: Relations between the elastic moduli and the plastic properties of polycrystalline pure metals. Philos. Mag. 45, 823 (1954)

73. Frantsevich, N., Voronov, F.F., Bokuta, S.A.: In: Frantsevich, I. N. (ed.) Elastic Constants and Elastic Moduli of Metals and Insulators Handbook, vol. 60. Naukova Dumka, Kiev (1983)

74. Schreiber, E., Anderson, O.L., Soga, N.: Elastic constants and Their Measurements. McGraw-Hill, New York (1973)

75. Harrison, W.A.: Electronic Structure and Properties of Solids. Dover, New York (1989)

76. Bouhemadou, A., Khenata, R., Kharoubi, M., Seddik, T., Reshak, A.H., Al-Douri, Y.: FP-APW+ lo calculations of the elastic properties in zinc-blende III-P compounds under pressure effects. Comput. Mater. Sci 45, 474 (2009)

77. Maachou, A., Aboura, H., Amrani, B., Khenata, R., Bin Omran, S., Varshney, D.: Structural stabilities, elastic and thermodynamic properties of scandium chalcogenides via first-principles calculations. Comput. Mater. Sci. 50, 3123 (2011)

78. Kleinman, L.: Deformation potentials in silicon. I. Uniaxial strain. Phys. Rev. 128, 2614 (1962)

79. Kim, K., Lambrecht, W.R.L., Segal, B.: Electronic structure of GaN with strain and phonon distortions. Phys. Rev. B 50, 1502 (1994)

80. Blackman, M. The specific heat of solids. Proc. R. Soc. Lond. A 1942; 181:58; ibid 1937; 159: 416; 1935; 149:126; 1935; 148:384; 1935; 148:365 
81. Blackman, M.: The specific heat of solids. Proc. R. Soc. Lond. A 159, 416 (1937)

82. Blackman, M.: The specific heat of solids. Proc. R. Soc. Lond. A 149, 126 (1935)

83. Blackman, M.: The specific heat of solids. Proc. R. Soc. Lond. A 148, 384 (1935)
84. Blackman, M.: The specific heat of solids. Proc. R. Soc. Lond. A 148, 365 (1935)

85. Gopal, E.S.R.: Specific heats at low temperatures. Plenum Press, New York (1966)

86. Tari, A.: The Specific Heat of Matter at Low Temperatures. Imperial College Press, London (2003) 